\title{
Dissection of protonation sites for antibacterial recognition and transport in QacA, a multi-drug efflux transporter
}

\author{
Puja Majumder $^{1}$, Shashank Khare ${ }^{1}$, Arunabh Athreya ${ }^{1}$, Nazia Hussain ${ }^{1}$, Ashutosh Gulati ${ }^{1,2}$, \\ Aravind Penmatsa ${ }^{1,}{ }^{*}$ \\ ${ }^{1}$ Molecular Biophysics Unit, Indian Institute of Science, Bangalore, India, 560012
}

\begin{abstract}
QacA is a drug: $\mathrm{H}^{+}$antiporter with 14 transmembrane helices that confers antibacterial resistance to methicillin-resistant Staphylococcus aureus strains, with homologs in other pathogenic organisms. It is a highly promiscuous antiporter, capable of $\mathrm{H}^{+}$-driven efflux of a wide array of cationic antibacterial compounds and dyes. Our study, using a homology model of QacA, reveals a group of six protonatable residues in its vestibule. Systematic mutagenesis resulted in the identification of D34 (TM1), and a cluster of acidic residues in TM13 including E407 and D411 and D323 in TM10, as being crucial for substrate recognition and transport of monovalent and divalent cationic antibacterial compounds. The transport and binding properties of QacA and its mutants were explored using whole cells, inside-out vesicles, substrate-induced $\mathrm{H}^{+}$release and microscale thermophoresis-based assays. The activity of purified QacA was also observed using proteoliposome-based substrate-induced $\mathrm{H}^{+}$transport assay. Our results identify two sites, D34 and D411 as vital players in substrate recognition, while E407 facilitates substrate efflux as a protonation site. We also observe that E407 plays an additional role as a substrate recognition site for the transport of dequalinium, a divalent quaternary ammonium compound. These observations rationalize the promiscuity of QacA for diverse substrates. The study unravels the role of acidic residues in QacA with implications for substrate recognition, promiscuity and processive transport in multidrug efflux transporters, related to QacA.
\end{abstract}

\section{Keywords}

MFS (Major facilitator superfamily); DHA (drug: $\mathrm{H}^{+}$antiport); MRSA (methicillin-resistant Staphylococcus aureus); MST (microscale thermophoresis); proteoliposome reconstitution

\footnotetext{
*Address for correspondence. Dr. Aravind Penmatsa, Assistant Professor, Molecular Biophysics Unit, Indian Institute Science, Bangalore, 560012, India. penmatsa@iisc.ac.in, Phone. +91-80-2293 2458. Fax+91-80-2360 0535; Email for strain requests abhijit@cdfd.org.in penmatsa@iisc.ac.in.

${ }^{2}$ Present address. Postdoctoral fellow, Dept. of Biochemistry and Biophysics, Stockholm University, Sweden.

Author Contributions. PM and AP designed the research; PM, SK performed the experiments with help from NH and AG; NH performed the ATPase purification, QacA reconstitution and transport assay. AA and AP carried out homology modeling and analyses; PM, AA and AP analyzed the data and wrote the manuscript.

Authors declare no conflicting interests.
} 


\section{Introduction}

Efflux of antibacterial compounds is a major mechanism of acquiring multi-drug resistance in many pathogens [1, 2]. Efflux transporters of the major facilitator superfamily (MFS) are one of the largest groups of proteins involved in proton-coupled antiport in several pathogenic strains of gram-positive and gram-negative bacteria [3]. In methicillin-resistant Staphylococcus aureus, chromosomally encoded antibiotic efflux pumps like NorA, NorB, NorC [4] and plasmid encoded antibacterial efflux pumps including QacA and QacB are involved in $\mathrm{H}^{+}$-driven transport $[4,5]$. MFS transporters involved in antiport consist of candidates with 12 or 14 transmembrane (TM) helices, referred to as the DHA1 and DHA2 families, respectively [6]. Among DHA1 members, crystal structures are available for Escherichia coli transporters MdfA, EmrD and YajR in different conformational states [7-9]. Extensive studies on MdfA and LmrP (Lactobacillus lactis) revealed diverse aspects of their functional properties involving substrate promiscuity [10], $\mathrm{H}^{+}$:drug stoichiometry and sites for substrate recognition [11, 12]. Members of the DHA1 family also comprise chromaffin granule and synaptic vesicle monoamine transporters (VMAT 1 and 2) that share mechanistic similarities and transport serotonin, dopamine and noradrenaline into vesicles using $\mathrm{H}^{+}$-gradient $[13,14]$. All DHA1 members previously studied have one or more acidic residues that are required for protonation and lipophilic cation transport $[12,15,16]$.

In contrast, with no available structures, the DHA2 family of antiporters have not been characterized extensively. QacA is a prototypical DHA2 member whose topology was determined to comprise 14 TM helices [17]. Proteins of the major facilitator superfamily have a conserved tepee-like architecture with two distinct six-TM helix bundles that are arranged with a pseudo-2-fold symmetry [18]. Harnessing this property, QacA was modeled based on the distantly related 14 TM helix crystal structures of prokaryotic protondependent oligopeptide transporters (POTs) ( 20\% seq. identity) [19, 20]. POTs are also observed to bind peptides using an "aromatic clamp" that was proposed in MdtM, a DHA1 member [21, 22]. In the 14TM POTs, the two additional TM helices are observed as an insertion between symmetric helical bundles 1-6 and 7-12. As a result, TM helices 1-6 and 9-14 (in DHA2) have a pseudo 2-fold symmetry that would allow rocking-switch movements to facilitate alternating access on either side of the membrane [23].

Unlike MdfA, QacA is capable of $\mathrm{H}^{+}$:drug stoichiometry of 2 or greater [24], and has an extensive substrate repertoire that includes nearly 30 organic monovalent and divalent cations, with antibacterial properties [25]. QacA is sensitive to inhibitors like verapamil and reserpine, of which the latter is a high affinity blocker of neurotransmitter transport, in both VMAT isoforms [24, 26]. A natural substitution of D323 to alanine is observed in QacB, a paralog of QacA with a corresponding inability to transport divalent substrates [17]. Antiporters possess at least one protonatable acidic residue in the TM regions where substrates compete and release protons in the opposite direction to the substrate flow [27]. However, it is frequently observed in transporters like LmrP [12], VMATs [28] and BbMAT [16], that more than one acidic residues exist within the transporter vestibule that can exchange two or more protons for every substrate molecule, rendering the process electrogenic [29]. 
Despite multiple studies on QacA's prevalence, substrate repertoire and topology, experimental evidence identifying residues critical for substrate recognition and protonation during transport process is lacking, presumably due to the absence of a representative DHA2 structure. In this study, we generated a homology model of QacA and identified six acidic residues in the vestibule, which could play a role in protonation-coupled binding and transport. To investigate their role in the substrate recognition and transport of structurally diverse monovalent and divalent cationic substrates, systematic mutagenesis of aspartate and glutamate residues in the vestibule of QacA was carried out. The substrates chosen for the study include monovalent compounds ethidium (Et), a cationic dye, tetraphenylphosphonium (TPP), and divalent compounds, pentamidine (Pm), a diamidine and dequalinium (Dq), a divalent quaternary ammonium compound. All the four compounds have previously been known to be substrates of QacA [24] and were chosen here for the diversity in their chemical structures. Substrate efflux was monitored using whole cell-based and inside-out vesicle-based transport measurements to understand the effect of mutations on the ability of QacA to transport them. We could also monitor the efflux activity of purified QacA reconstituted alongside $\mathrm{F}_{0} \mathrm{~F}_{1}$ ATPase using substrate-induced proton transport assay. Using purified QacA and its mutants, we further deduced the important roles of individual sites through substrate-induced proton release and direct-binding measurements using microscale thermophoresis (MST).

We identify that substrate recognition occurs at two distinct sites of the transporter, at D34 (TM1) and D411 (TM13), both of which are vital for binding and transport. In addition, we observe that E407 is a likely protonation site that facilitates efflux. Rather interestingly, for a dicationic drug Dq, D411 ceases to be the second recognition site and its role is replaced by E407, thereby providing additional insights into the promiscuous abilities of QacA to bind and transport diverse substrates using discrete sets of substrate recognition sites.

\section{Results}

\section{Homology model reveals six acidic residues in the vestibule of QacA}

A 14TM homology model of QacA was built using prokaryotic POT structures [30] and MdfA as well as several other crystal structures as templates, using I-TASSER (Fig. 1a) [31]. QacA sequence lacking the region 432-474, corresponding to extracellular loop-7 (connects TM 13 and 14), was used for the modeling study. The topology of QacA model, with the highest score, resembles POTs, wherein TMs 1-6 and TMs 9-14 have inherent pseudo- 2 fold symmetry and TMs 7 and 8 form an insertion that connects the two domains that undergo alternating-access (Fig. S1) [23]. The boundaries of the modeled TM helices broadly adhere to the previously determined experimental topology [17]. The model exhibits an inward-open conformation with solvent accessibility toward the cytosolic compartment. We observed six acidic residues, lining the vestibule of QacA at different levels including D34 (TM1), D61 (TM2), D323 (TM10), E406 (TM13), E407 (TM13) and D411 (TM13) (Figs. 1a, b and S1). With the exception of D323 and E406, each of the acidic residues is well conserved among closely related QacA homologs (Figs. 1b and S2). The accessibility of individual residues was verified by mutating each of them to a cysteine. The absence of cysteine residues in the primary sequence of QacA facilitated our analysis of accessibility to 
acidic residues within the vestibule, using polyethylene glycol-maleimide (PEG-Mal, $5 \mathrm{kDa}$ ) that covalently binds to free thiol groups [32]. Single-cysteine substitutions of QacA at E406, E407 and D411 exhibited a greater propensity for PEG-Mal modification-induced gel shifts, whereas substitutions at D34, D61 and D323 exhibited minimal labeling, suggesting reduced PEG-Mal accessibility (Fig. 1c). The greater accessibility of PEG-Mal to residues toward the cytosolic part of the transporter vestibule suggests that QacA exists predominantly in the inward-open conformation in detergent micelles and the homology model reflects this orientation. However, the labeling propensity observed in this experiment does not address the likelihood that mutation of an acidic residue could affect conformational equilibrium of the transporter.

We further evaluated the model by exploring the functional importance of the region corresponding to the TM helices 7 and 8 by deleting a stretch of residues from 209 to 258 . Despite the deletion, the protein expression displayed a homogenous profile as observed using fluorescence-detection size exclusion chromatography (FSEC) and a predominantly ahelical circular dichroism spectra, suggesting structural integrity (Fig. S3). However, the deletion construct suffered a complete loss of function as observed using survival assays and a significant loss of binding affinity toward TPP (Fig. S3d) in comparison to the wild-type (WT) QacA. The observation suggests that TMs 7 and 8 in QacA play a significant role in mediating efflux, in accordance with the observations with Tet(L) TM 7 and 8 deletion that loses the ability to provide tetracycline resistance [33]. The ability of WT QacA to transport drugs was tested at high extracellular $\mathrm{pH}(8)$, where the $\mathrm{pH}$ gradient is insignificant across $E$. coli membranes (Fig. 2). E. coli expressing WT QacA could robustly protect cells against TPP, Pm and efflux Et when the extracellular $\mathrm{pH}$ was 6, whereas this ability was compromised when the extracellular $\mathrm{pH}$ was enhanced to $\mathrm{pH} 8$, suggesting that transport in QacA is driven primarily by $\mathrm{pH}$ gradient and to a lesser extent through membrane potential.

Proton-driven transport of positively charged or polar molecules through QacA requires acidic residues inside the vestibule that are capable of reversible protonation upon lipophilic cation interactions. The significance of acidic residues observed within the QacA vestibule was characterized through individual residue substitutions to alanine and neutral substitutions to asparagine or glutamine. Constructs with and without a GFP-His8 tag at the C-terminus were built to facilitate expression analyses through FSEC and Western blots, respectively (Fig. S4).

\section{Expressed QacA is active in native membranes}

Prior to assessing the roles of individual residues in transporting the cationic substrates, WT QacA was assayed for transport activity and substrate recognition, in whole cells, inside-out vesicles and as detergent isolates for its behavior in the same experiments. Capacity to transport Et was checked in whole cell-based efflux assay, where a relative decrease in the fluorescence of Et was correlated with its efflux from energized cells. To assess the transport properties of QacA in case of TPP, Pm and Dq, inside-out vesicles were prepared and 9amino-6-chloro-2-methoxyacridine (ACMA) was used as a $\mathrm{pH}$ gradient sensing fluorescent probe. Dequenching of ACMA fluorescence was used as a measure of substrate-induced proton antiport (Fig. 3a). 


\section{Purified QacA actively interacts with substrates and can transport substrates in reconstituted liposomes}

QacA was purified as described earlier [34] using affinity purification with a moderate yield and high level of purity (Fig. S5a), using undecyl- $\beta$-D-maltopyranoside (UDM) after screening through multiple non-ionic detergents. Purified QacA displays a time and concentration dependent formation of a higher oligomeric species in solution whose molecular mass corresponds to a QacA trimer. When the protein was concentrated beyond 1 $\mathrm{mg} / \mathrm{ml}$ there was a tendency to form aggregates, and this precluded structural and biophysical studies requiring concentrated samples of QacA. The binding and competition studies performed with purified QacA were therefore carried out in dilute samples where QacA displays a predominantly monomeric species.

Binding of substrates to the purified QacA was evaluated using MST that allowed rapid estimation of binding affinities (Fig. 3b). The binding affinity obtained through MST was validated using fluorescence quenching of QacA with TPP addition that gave values similar to MST data (Fig S5b, Table 1). This validation gave us the confidence to pursue MST as a rapid tool for estimation of binding affinities with other substrates and mutants. The affinities we report for QacA-substrate interactions are lower than what is generally observed with other DHA members. For instance, MdfA has an affinity of $4.7 \mu \mathrm{M}$ for TPP [35], whereas QacA displays a weaker affinity $\left(K_{\mathrm{d}}\right)$ of $\sim 350 \mu \mathrm{M}$. We speculate that the lowered affinity could be a result of the extraction of QacA into detergent micelles, which may have partially compromised the ability of substrates to partition and get locally enriched in the lipid bilayer and enter the transporter directly through the membrane, as suggested in case of LmrP [36, 37]. This could serve as a plausible rate-limiting step for binding of antibacterials to detergent extracted QacA, while retaining micromolar enzyme activity $\left(K_{\mathrm{M}}\right)$ in its native membrane environment [24].

In substrate-induced proton release assays, the unbuffered solution of purified QacA was acidified followed by a step-wise addition of fixed concentrations of the substrate [22]. The release of protons induced by substrate binding was monitored through quenching of a $\mathrm{pH}$ sensitive probe fluorescein (Fig. 3c). MST experiment and substrate-induced proton release assay display that purified QacA can actively interact with its substrates. In order to test the transport activity, purified WT QacA was reconstituted alongside E.coli $\mathrm{F}_{0} \mathrm{~F}_{1}$ ATPase to facilitate $\mathrm{pH}$ gradient formation in response to the addition of ATP (Fig. 4a, b). The acidification of the liposomes was tested using ACMA fluorescence that undergoes quenching with increased $\mathrm{pH}$ gradient across the liposome membrane, in an assay that is akin to the inside-out vesicles used to demonstrate transport activity of QacA and its mutants. In QacA containing proteoliposomes, the addition of a substrate must induce dequenching of the ACMA fluorescence due to the antiport activity resulting in substrateinduced proton transport across the membrane. Valinomycin was retained throughout the experiment to avoid the buildup of membrane potential. Dequenching of ACMA fluorescence was clearly observed by the addition of all three substrates TPP, Pm and Dq, whereas an equivalent amount of substrate's solvent addition did not give any dequenching of ACMA fluorescence (Fig. 4c). A control experiment done using proteoliposomes with ATPase alone without QacA, showed that presence of QacA induces a substantial 
enhancement of substrate-induced $\mathrm{H}^{+}$release as compared to leaks caused by addition of substrate (Fig. 4c). The experiment unambiguously establishes the ability of QacA to display antibacterial-induced proton transport, upon reconstitution.

\section{Acidic residues in the vestibule exhibit variable importance to counter drug resistance}

The alanine and asparagine/glutamine mutants at individual sites were tested alongside WT QacA for toxicity upon overexpression, and none of the mutants displayed a toxic phenotype in the E.coli strain JD838 upon induction (Fig. S6a). A control mutation performed away from the vestibule, in the extracellular loop-7, D434N, had a significantly lowered expression (Fig. S6b). However, despite the minimal expression, no effect was observed in the drug resistance assays performed using Et, TPP, Pm and Dq, in comparison with WT QacA. FSEC was used to rapidly analyze expression levels and homogeneity of QacA-GFP and its mutants (Fig. S4a-f). All the mutants exhibited detectable expression in the range of 44\%-125\% in comparison to the WT (Table S1). Expression level of QacA mutants, in case of experiments done with QacA-His construct in native membranes, was evaluated using Western blots (Fig. S4g). The cells expressing these mutants were tested for their ability to grow at different dilutions using monovalent cationic substrates including Et, TPP and divalent cationic substrates including Pm and Dq (Fig. 5a). Due to the absence of three major efflux transporter genes $m d f A, a c r B$ and $y d h E$, the JD838 strain is highly susceptible to small doses of antibacterial compounds. Experimentally optimized concentrations of substrates were used to perform the assay at neutral pH. QacA single mutants at D34 lost their ability to survive, irrespective of the cationic compounds tested. This was followed by loss of activity with single mutants at D411 with most drugs, except Dq (Fig. 5b). In addition to D34, E407 mutants also displayed a loss of survival with Dq as the antibacterial, instead of D411, whereas the survival of other mutants remained mostly unaffected in the presence of the drug. Also, the survival of D323 mutants was unaffected by Dq despite being implicated in dicationic drug efflux, a phenomenon attributed to processive transport in an earlier study [38]. However, Pm had a pronounced effect on cell survival with minimal growth observed in most of the mutants including D323. We also observed compromised survival in D61 and E406 mutants in case of Et, TPP and Pm, although subsequent transport studies displayed retention of transport activity despite mutagenesis. Based on this observation, we retained our focus on testing individual sites that had a near-complete loss of transport activity. The findings thereby raise the interesting possibility of a few crucial sites essential for survival against antibacterial stress and distinct subsets of acidic residues required for transport of drugs with different chemical structures. In multiple instances, mutations in acidic residues yielded minimal growth in the survival assay, although their ability to bind and transport antibacterial compounds was ascertained directly, as described in the subsequent sections.

\section{Substrate recognition occurs at two sites in QacA}

To analyze the roles of individual residues for substrate recognition and transport, we conducted whole cell-based Et efflux assays (Fig. 6), inside-out vesicle-based assays (Figs. 7, S7 and S8), substrate-induced proton release assays (Figs. 8, S9) and affinity studies (Table 1, Fig. S10) in mutant backgrounds, with TPP, Pm and Dq. The alanine and neutral substitutions at individual residues were analyzed for changes in transport properties of 
QacA. Neutral substitutions of aspartate (asparagine) and glutamate (glutamine) are suggested to mimic permanently protonated forms [39, 40], where the substrate cannot compete for the negative charge by displacing protons [39, 41]. A significant outcome of these assays is the characterization of D34 and D411 as essential sites for substrate recognition, due to which we observed a severe loss in transport activity for most lipophilic cations used in this study (Figs. 6a, 6f and 7). D34 was predicted as analogous to E26 residue in the TM1 of MdfA [15] and D33 of VMAT [42], but its role has not been investigated in earlier reports on QacA. Neutral substitution at D34 also compromised the ability of substrates to compete for protonation sites resulting in a near-complete loss of proton release as observed with TPP or retaining a minimal ability to compete for protons as observed in Pm and Dq (Fig. 8b). This loss of phenotype may stem from loss of measurable binding interactions with D34N QacA mutant as the dissociation constants could not be measured for any of the three drugs (Table 1, Fig S10).

Interestingly, we found that D411 (TM13), topologically present toward the cytosolic end of the molecule, is also necessary for cells' survival against all substrates, except Dq (Fig. 5). Efflux experiments for Et, TPP and Pm indicate a crucial role of D411 in the activity of QacA, with both alanine and asparagine mutants displaying compromised activity for all three substrates (Figs. 6f and 7), whereas the ability of D411A/N mutants to release protons induced by Dq remains unaltered in comparison to WT (Figs. 7c and 8c). The interaction propensities also reflect in the binding of TPP and Pm whose affinity to D411N could not be determined. However, Dq retains similar binding affinities with D411N and WT $(0.71 \mathrm{mM}$ versus $0.9 \mathrm{mM}$ ) (Table 1, Fig. S10), signifying the role of D411 as an important secondary substrate recognition site, specific to monovalent cations and divalent cationic substrates like Pm, but not Dq.

The presence of two distinct substrate recognition sites in QacA located in both cytosolic and extracellular halves of the transporter provides additional anchoring sites for the substrates to interact with, during the transport process. The finding that QacA employs different residues to recognize $\mathrm{Dq}$ as opposed to monovalent cations and $\mathrm{Pm}$ provides interesting hints into the promiscuity of substrate recognition that is explored in the subsequent section.

\section{D323 is required for Pm transport but not for other long-divalent cations}

QacB, a paralog of QacA, has an alanine at the position 323 that was previously observed to impair transport of divalent substrates like Pm and propamidine [24]. However, recent observations suggest that it retains the ability to transport some divalent cationic drugs, particularly the ones separated by a long linker including Dq and chlorhexidine (Cx) [38]. As seen in the survival assays, QacA D323A/N mutants confer a stark contrast in the survival phenotype of the cells grown in the presence of monovalent cations versus $\mathrm{Pm}$. While cells harboring D323A/N mutants survive well in the presence of Et and TPP, the ability to survive was lost in case of Pm, while in the presence of Dq, it remained unaltered (Fig. 5). Dq transport across inside-out vesicles remains active despite mutations at D323, indicating its importance specifically for Pm transport (Figs. 7b and c). A similar phenomenon was observed also with $\mathrm{Cx}$ where $\mathrm{D} 323 \mathrm{C}$ mutation did not affect cell survival 
[38]. Also, with QacA D323N, the ability of the substrate to compete for protons in solution does not change with TPP and Dq in comparison with WT QacA (Fig. 8a and d). In line with the earlier experiments, Pm loses its ability to release protons from QacA D323N with a reduction in the number of $\mathrm{H}^{+}$- release steps, as compared to WT, prior to saturation. The importance of D323 for primarily interacting with Pm is also reflected in the binding affinities obtained for D323N, which binds to TPP and Dq with affinities comparable to WT QacA ( $K_{\mathrm{d}}$ of $\sim 0.3 \mathrm{mM}$ for TPP and $\sim 0.5 \mathrm{mM}$ for Dq) whereas Pm shows a clear loss of affinity for the D323N mutant (Table 1, Fig. S10). The results indicate that D323 is not a crucial determinant of QacA's ability to transport divalent cationic drugs but is important for specific substrates of QacA like Pm and likely propamidine [24].

\section{E407 plays a dual role in substrate transport}

E407 seems unique among the group of residues, as it is the only site whose loss of transport phenotype upon mutation to alanine is rescued by its neutral mutation to glutamine. E407Q displays a mild recovery in survival in comparison to E407A and its efflux properties with Et, TPP and Pm are comparable to WT QacA (Figs. 5, 6e and 7a and b) whereas E407A is incapable of transporting all three compounds. Interestingly, E407Q displayed a reduced number of titration steps by both TPP and Pm in comparison to WT QacA (Fig. 8e). Also, the binding properties of E407Q indicate a reduction in binding of TPP ( $K_{\mathrm{d}}$ of $\left.1.65 \mathrm{mM}\right)$ whereas Pm affinity remains largely unaltered $(K \mathrm{~d}$ of $0.58 \mathrm{mM})$ in comparison to WT (TPP: $K_{\mathrm{d}}$ of $0.36 \mathrm{mM}, \mathrm{Pm}: K_{\mathrm{d}}$ of $1 \mathrm{mM}$ ) (Table 1, Fig. S10), implying that it plays a minimal role as a substrate recognition site, although it is needed for the survival of the host. Our observations indicate the likelihood that E407 acts as a protonation site in QacA given the ability of neutral substitutions of acidic residues to uncouple transport from $\mathrm{pH}$ gradients [41]. Despite the proximity of E406 to E407 and compromised survival of E406 mutants in comparison to WT QacA, E406A/Q mutants are capable of transporting all four substrates used in the study, suggesting that the effects caused by E407 are indeed specific and cannot be substituted by neighboring residues.

While E407 serves as a potential protonation site for TPP and Pm (Fig. 9), in the case of Dq, E407 is essential for substrate recognition. The survival assays in the presence of Dq demonstrate that cells harboring E407A/Q mutants fail to grow whereas D411A/N mutants exhibit normal growth (Fig. 5). This apparent swap in their roles with respect to Dq strongly supports the promiscuity observed in the substrate repertoire of QacA, given that these residues are only one helical turn apart. In such a case, it would be plausible to argue that E407 acts as a substrate recognition site for one of the two charged moieties of Dq. This is supported by a range of observations, including rescue of phenotype observed in the background of E407Q mutant in the proton release driven by Et, TPP and Pm but absence of Dq efflux irrespective of A or Q substitution at E407 (Figs. 6e and 7). E407Q mutant has a similar affinity for Pm ( $K_{\mathrm{d}}$ of $1.0 \mathrm{mM}$ in WT versus $K_{\mathrm{d}}$ of $0.58 \mathrm{mM}$ in E407Q) and reduced affinity for TPP ( $K_{\mathrm{d}}$ of $0.36 \mathrm{mM}$ in WT versus $K_{\mathrm{d}}$ of $1.65 \mathrm{mM}$ in E407Q), but is completely lost for Dq (Table 1, Fig. S10).

We made two double amino acid substitutions in QacA, namely, D34N/E407Q and D34N/ D411N to evaluate the effect on substrate-induced $\mathrm{H}^{+}$-release (Fig. $8 \mathrm{f}$ and g). In these 
combination mutants, TPP- induced proton release was not observed with either of the two mutants since the D34N substitution alone was debilitating for TPP-induced $\mathrm{H}^{+}$release. Pm could induce limited proton release in D34N/E407Q combination, attributing it to an unaltered D411. Dq, on the other hand, did not seem to interact with any of the mutant combinations, likely owing to the important role played by D34 for the same.

The results strongly support the notion of discrete sets of residues being important for recognition and transport of antibacterial substrates of QacA. The essential residues are characterized by an "all-or-none" effect on substrate interaction and transport properties with single site mutations. These previously uncharacterized roles of acidic residues in QacA vestibule could be applicable to related DHA2 transporters, due to the strong conservation observed in the identified sites. The study also identifies a hitherto unknown region at the cytosolic part of TM13 as a hotspot for multi-substrate recognition and protonation driven transport, in DHA2 members.

\section{Discussion}

Mechanistic studies on multi-drug efflux transporters suggested the importance of acidic residues as the primary determinants of substrate recognition and transport [43, 44], although this aspect has remained unexplored in QacA, a promiscuous multi-drug efflux transporter [25]. This study dissects the roles of acidic residues in the vestibule of this transporter through mutational analyses, aided by a molecular model, providing hitherto unanticipated insights into their roles in QacA's substrate-specific efflux properties. Among the six acidic residues, four residues D34 (TM1), D323 (TM10), D411 (TM13) and E407 (TM13) were seen to be crucial for promiscuous substrate recognition and translocation (Table 2).

Earlier studies involving DHA1 members including MdfA, LmrP and BbMAT have highlighted the important roles that protonatable acidic residues play in substrate recognition and $\mathrm{H}^{+}$:drug stoichiometry[12, 16, 43]. Asp and/or Glu residues surrounding the binding pocket are protonated and act as essential sites to trigger rocking-switch conformational changes that allow cationic substrates to compete and release protons in the transport cycle[27, 36]. Alternately, protonation of additional sites in the vestibule enhances the $\mathrm{H}^{+}$: drug stoichiometry and renders the process electrogenic [44]. The characteristic features of each of these sites can be evaluated through substitutions of individual residues. Mutations at protonation sites involved in substrate recognition leading to competitive proton release, result in significant loss of interaction propensities and transport activity. On the other hand, substitutions at protonation sites that facilitate electrogenic transport retain binding propensities but have compromised substrate translocation. Protonation mimicking substitutions at these sites can recover transport albeit with a reduced ability to couple proton gradients to substrate efflux [45]. Numerous studies, particularly on MdfA and LmrP, were used to evaluate the importance of protonatable acidic residues $[10,12,16]$ leading to some rather interesting observations. For instance, in MdfA, relocation of the protonatable acidic residue E26 from TM1 to TM10 (V335E) was observed to retain efflux properties. Also, introduction of a negative charge (G354E) in TM11 allows the conversion of MdfA's 
efflux property from electroneutral to electrogenic [43]. Incidentally, the position of G354E mutation in MdfA coincides with the position of E407 in TM13 (Fig. S1c) in QacA.

We observe that QacA naturally encompasses many of these functional determinants reported in DHA1 members. Among the six acidic residues that were explored, D34 stands out as an essential antibacterial recognition site, irrespective of the substrate used in the study. This conserved site is akin to the substrate recognition sites of MdfA (E26), VMAT (D33) and BbMAT (D25) and is located in TM1[15, 16, 42]. Substitution of D34 to alanine or asparagine was not tolerated and resulted in completely compromised binding and transport properties. The substrate-induced $\mathrm{H}^{+}$release experiments performed with QacA D34 $\mathrm{N}$ mutant did not elicit any $\mathrm{H}^{+}$-release upon addition of monovalent or divalent cations, thereby reinforcing the importance of D34 as an essential substrate recognition site vital for QacA's efflux activity. Despite its importance, we observe that D34 displays minimal accessibility to PEG-Mal (5 kDa) crosslinking in detergent extracted QacA. However, the residue could still be accessible to smaller sized lipophilic cations (300-500 Da) as seen in the case of the rhodamine $6 \mathrm{G}(\mathrm{R} 6 \mathrm{G})$ bound crystal structure of $\mathrm{Na}^{+} / \mathrm{H}^{+}$coupled multidrug and toxin extrusion (MATE) transporter, DinF (PDB ID 4LZ9). This structure revealed that rhodamine $6 \mathrm{G}$ could directly interact with the substrate recognition site, D40, in a cleft despite its minimal solvent accessibility [46]. The added advantage for a substrate recognition site with reduced solvent accessibility is the increase in pKa [47] that translates to an easier competition of $\mathrm{H}^{+}$ions with the interacting antibacterial compounds, at neutral $\mathrm{pH}$.

QacA is known to have a $\mathrm{H}^{+}$:drug stoichiometry of 2 or greater [24] and our observations have highlighted the role of additional acidic residues that aid in antibacterial efflux in this electrogenic transporter. A bulk of these acidic residues cluster around the cytosolic half of TM13 and line the solvent accessible vestibule. Among the three acidic residues observed here (E406, E407 and D411), E406 is not essential for transport (Table 2). On the other hand, D411 is critical for the transport of Et, TPP and Pm as QacA D411A/N mutants lose their ability to translocate the three substrates. Compromised binding and substrate-induced proton release were observed with TPP and Pm in the D411N mutant suggesting the importance of this residue in the recognition of these substrates. Intriguingly, mutations at D411 have no influence on the ability of QacA to interact and transport Dq. We, therefore, consider the role of D411 as conditional, suggesting its importance in the efflux of a subset of substrates, instead of being important for all of them. Alternately, QacA E407A/Q mutants had a dissimilar but essential role in substrate efflux, in comparison with D34 or D411. We note that the E407A mutant completely lost the ability to transport Et, TPP and Pm but remarkably, the E407Q substitution retains transport activity. It is of interest to note that numerous studies involving ion channels [40], DHA members [36] and MATE transporters [46], have employed neutral substitutions of acidic residues to mimic permanent protonation. The retention of transport activity with E407Q suggests the residue's role as a likely protonation site in QacA, with the mutation retaining the transport but uncoupling the dependence of substrate efflux on $\mathrm{pH}$ gradient. Uncoupling of substrate flux from ion gradients leads to leaky transport and functional shifts in transport properties. For instance, substitutions of acidic residues lead to leaky transport in Mal11 (E167, D123) [48] and uncoupling of Na+-dependence in NorM (D367N), a MATE transporter for Et efflux [49]. 
In alignment with its role as a protonation site, the E407Q mutant also retains normal binding propensities toward both TPP and Pm. However, when it comes to the transport of Dq, we report a rather interesting shift in the role of E407 residue wherein it ceases to be an additional protonation site for Dq transport, instead acting as a substrate recognition site. Our experiments clearly reveal that the QacA E407Q mutant lacks the ability to interact or transport Dq. The differences in site specificities for antibacterials like Pm and Dq could result from variations in the length of the linker that separates the cationic charges within the divalent substrates. Dq with a longer linker is likely accommodated with its second cationic charge interacting with E407 instead of D411 which is located one helical turn above E407 (Fig. 9). This selective difference in interactions based on the characteristics of the transported substrate is a testimony of QacA's promiscuous substrate recognition properties. Put together, these observations suggest that E407 is an essential site that can take up multifaceted roles of a protonation site for some antibacterial compounds or serve as a substrate recognition site for specific substrates like Dq.

Earlier studies on QacA attributed the ability to transport divalent cations to a single substitution D323A observed in QacB, a paralogue of QacA, with a consequent loss of transporting divalent cationic drugs [17]. However, in recent studies including ours, this poorly conserved residue was observed to retain the ability to transport multiple divalent cationic antibacterials including Dq and $\mathrm{Cx}$ [38]. The ability to transport long divalent cations was attributed to processive transport in MdfA and QacA D323C mutant [38]. We, however, do see heavily compromised Pm binding and transport suggesting the importance of D323 as a site with conditional importance for a narrow spectrum of antibacterial compounds.

Taken together, our analyses of the acidic residues in QacA highlight the roles of four important sites (Fig. 9). These results establish that D34 is essential for antibacterial efflux as a substrate recognition site followed by D411 that is required for maintaining QacA efflux for a subset of substrates but is of no functional consequence for Dq. E407 can assume a dual role of a protonation site for most substrates but in case of Dq, it plays the role of a substrate recognition site. D323 is non-essential for translocation of monovalent cations, Dq and Cx [38] but is important for the efflux of Pm like antibacterials. This study identifies a cluster of well conserved acidic residues in TM13 that could potentially aid as secondary interaction sites during processive transport implicated as a mechanism of efflux for long dicationic substrates [38].

While this study reveals the roles of individual residues in promiscuous antibacterial recognition and efflux in QacA and related members, further studies that probe the conformational shifts in the transporter in response to changes upon substrate interactions, $\mathrm{pH}$ differences and mutational effects would highlight the steps in the transport cycle of QacA. This would be greatly aided by structural snapshots of QacA in multiple conformations that will also facilitate strategies toward the design of specific efflux pump inhibitors against this class of drug efflux transporters. 


\section{Methods}

\section{Plasmids and Strains}

JD838 ( $\Delta m d f A \Delta a c r B \Delta y d h E:$ Kan) strain is a derivative of the E. coli K-12 strain, LMG194, bearing a knockout of 3 multi-drug efflux pumps. A codon optimized QacA synthetic gene [34] was cloned into pBAD-His 8 vector between $\mathrm{NdeI}$ and HindIII sites. CGFP construct was generated using megaprimer based whole plasmid PCR method. Site directed mutagenesis was performed using individual mutant primers and confirmed by DNA sequencing. The mutants of QacA include D34A/N, D61A/N, D323A/N, D411A/N, D434A/N, E406A/Q, E407A/Q, D34N/E407Q and D34N/D411N. E. coli DK8 strain ( $\Delta u n c B-C$, ilv::Tn 10) harboring plasmid pBWU13 (with wild type unc operon)[50] was used for ATPase purification.

\section{Homology model and sequence analysis}

The primary sequence of QacA $\Delta 432-474$ construct was modeled through homology modeling on I-TASSER server [31]. Known structures of homologous proteins and secondary structure restraints were used to thread the helices while a known structure of a POT transporter (PDB ID: 4IKV) was used to model QacA. One of the output structures that satisfied the structural characteristics of MFS transporter was chosen. The deleted region from residue no. 432-474 was built as a loop in the existing model using Completionist [51]. The model was embedded in a POPC bilayer made using CHARMM-GUI (http:// www.charmm-gui.org) and was energy minimized using charmm $36 \mathrm{~m}$ force-field [52]. To check the stability of the model, the whole system was simulated for $20 \mathrm{~ns}$ in explicit solvent using Gromacs 5.1.4 package [53]. As the Ca RMSD stabilized, one frame was selected as the final model from the trajectory.

\section{Accessibility assay}

WT QacA lacks cysteine residues in its sequence. Hence, the accessibility of acidic residues present in the vestibule to PEG-Mal $(5 \mathrm{kDa})$ was checked by generating cysteine mutants of D34, D61, D323, E406, E407 and D411 systematically. The proteins purified in phosphate buffer at $\mathrm{pH} 7$ were incubated with PEG-Mal $(0,100,250 \mu \mathrm{M})$ for $30 \mathrm{~min}$ at room temperature, followed by addition of $1 \mathrm{mM}$ of $\beta$-mercaptoethanol for $5 \mathrm{~min}$ and incubation at room temperature to chemically inactivate excess PEG-Mal. The samples were loaded onto a 12\% SDS-PAGE along with similarly treated but chemically unmodified WT QacA as a control. The experiment was repeated twice.

FSEC

Cells induced for expression were lysed by sonication and the protein was extracted using 20 mM UDM. The debris was pelleted down by centrifugation at 16000g. The supernatant was taken and run in FSEC through a Superdex 200 increase column $\left(\lambda_{\mathrm{Ex}}=488 \mathrm{~nm}\right.$ and $\lambda_{\mathrm{Em}}=510 \mathrm{~nm}$ ). Expression was quantitated using the area under the curve, and normalized with respect to expression of CGFP construct of WT-QacA. 


\section{Drug resistance assay}

Resistance to the cationic compounds (Et, TPP, Pm and Dq) was assessed using JD838 cells expressing WT QacA and its alanine or neutral mutants. For experiments on solid medium ( $2 \% \mathrm{w} / \mathrm{v} \mathrm{LB}$ and $1.8 \% \mathrm{w} / \mathrm{v}$ agar), cells were diluted to an $\mathrm{OD}_{600}$ of 1.0 , and $2 \mu \mathrm{l}$ of a series of a series 10 -fold dilutions were spotted on LB plates containing $0.05 \%(\mathrm{w} / \mathrm{v}) \mathrm{L}$-arabinose and $100 \mu \mathrm{g} / \mathrm{ml}$ ampicillin with or without the addition of the toxic cationic substrates ( $25 \mu \mathrm{M}$ of Et, $12 \mu \mathrm{M}$ of TPP, $12 \mu \mathrm{M}$ of Pm and $10 \mu \mathrm{M}$ of Dq). To check for the resistance against the toxic substance, the growth was analyzed after $12 \mathrm{~h}$ incubation at $37^{\circ} \mathrm{C}$. Similarly treated empty plasmid containing cells were used as a control. The assay was performed three times independently.

\section{Preparation of inside-out vesicle}

JD838 cells expressing WT QacA or its mutants were grown for $12 \mathrm{~h}$ at $20^{\circ} \mathrm{C}$ in the presence of $0.05 \%(\mathrm{w} / \mathrm{v}) \mathrm{L}$-arabinose and $100 \mu \mathrm{g} / \mathrm{ml}$ ampicillin. Cell were harvested and washed with $50 \mathrm{mM}$ potassium phosphate buffer $(\mathrm{pH} 7)$ and resuspended in the same buffer containing $1 \mathrm{mg} / \mathrm{ml}$ of lysozyme. After $30 \mathrm{~min}$ of incubation at $30{ }^{\circ} \mathrm{C}$, cells were broken with 5-8 passes at $\sim 500$ to 600 bar pressure using a high-pressure homogenizer. The crude membranes were incubated for $30 \mathrm{~min}$ at $30^{\circ} \mathrm{C}$ with $10 \mathrm{mM} \mathrm{MgSO} 4$ and $10 \mu \mathrm{g} / \mathrm{ml}$ of DNase I. Unbroken cells and cell debris were removed by centrifugation at $13000 \mathrm{~g}$ for $10 \mathrm{~min}$ at 4 ${ }^{\circ} \mathrm{C}$. Inside-out membrane vesicles were then isolated by ultracentrifugation at $125000 \mathrm{~g}$ for 1 $\mathrm{h}$ at $4{ }^{\circ} \mathrm{C}$ and resuspended in $50 \mathrm{mM}$ potassium phosphate buffer (pH 7) and $10 \%(\mathrm{v} / \mathrm{v})$ glycerol. Vesicles were then frozen in liquid nitrogen and stored at $-80{ }^{\circ} \mathrm{C}$ for further use.

\section{Substrate-induced $\mathrm{H}^{+}$transport in inside-out vesicles}

Inside-out vesicles were prepared as mentioned above. The frozen vesicles were thawed quickly for $50 \mathrm{~s}$ at $46^{\circ} \mathrm{C}$ for the assay. Each measurement was done with $24 \mu \mathrm{g}$ of total protein (estimated with Bradford method). Level of expression of each His-tagged construct was checked by Western using a mouse anti-His antibody conjugated to horseradish peroxidase (Sigma) and developed using Clarity chemiluminescence substrate (Bio-rad) (Fig. S4g) with purified QacA as positive control. The inside-out vesicles were diluted in a 2 $\mathrm{ml}$ solution of $50 \mathrm{mM} \mathrm{KCl}$ and $10 \mathrm{mM} \mathrm{MgSO}_{4}$. In the beginning of the experiment, $4 \mu \mathrm{M}$ ACMA and $0.5 \mu \mathrm{M}$ valinomycin were added and incubated for $5 \mathrm{~min}$. The samples were continuously stirred during measurement of ACMA fluorescence $\left(\lambda_{\mathrm{Ex}}=409 \mathrm{~nm}, \lambda_{\mathrm{Em}}=474\right.$ $\mathrm{nm}$ ) using a Fluoromax-3 (Horiba) fluorescence spectrophotometer. ATP $(100 \mu \mathrm{M})$ was added at $50^{\text {th }}$ second to create $\mathrm{pH}$ gradient required for transport process of QacA. As the gradient was established at $250^{\text {th }}$ second, $1 \mathrm{mM}$ substrate (TPP, Pm or Dq) was added externally and the change in $\mathrm{pH}$ due to transport of the substrate was measured. The reaction was terminated using $4 \mu \mathrm{M}$ nigericin at $300^{\text {th }}$ second. The assay was performed with two independent batches of inside-out vesicles.

\section{Whole cell efflux assay}

For measuring Et efflux, JD838 cells were grown at $37^{\circ} \mathrm{C}$ for $3.5 \mathrm{~h}$ in the presence of 100 $\mu \mathrm{g} / \mathrm{ml}$ ampicillin and $0.05 \%(\mathrm{w} / \mathrm{v}) \mathrm{L}$-arabinose. After the cells were washed three times and resuspended in $20 \mathrm{mM}$ Hepes (pH 7), $50 \mu \mathrm{M} \mathrm{EtBr}$ was added and incubated with for $1 \mathrm{~h}$ at 
$37^{\circ} \mathrm{C}$ in presence of $0.5 \mu \mathrm{M}$ carbonyl cyanide m-chlorophenyl hydrazone. Cells loaded with Et were washed three times with the same buffer. D-glucose $(5 \mathrm{mM})$ was used to energize the cells. The efflux assay $(\lambda E x=530 \mathrm{~nm}$ and $\lambda E m=610 \mathrm{~nm})$ was done using Varioskan Flash (Thermo Scientific) plate reader with intermittent shaking. The assay was done in triplicate.

\section{QacA protein purification}

Membrane preparation was done using standard protocol. The membrane was homogenized in $30 \mathrm{mM}$ phosphate buffer $(\mathrm{pH} 7)$ and $0.5 \mathrm{mM}$ PMSF (Protease inhibitor) was added during homogenization. UDM $(20 \mathrm{mM})$ was used for protein extraction from the homogenized membrane by incubating with it for $1 \mathrm{~h}$. Ultracentrifugation was done at $\sim 125000 \mathrm{~g}$ and the solubilized protein was present in the supernatant. Ni-NTA resin (pre-equilibrated in the buffer) was used for binding by nutating with it for $1 \mathrm{~h}$ at $4{ }^{\circ} \mathrm{C}$. The resin bound to protein was washed [with $30 \mathrm{mM}$ Phosphate buffer (pH 7), $120 \mathrm{mM} \mathrm{NaCl}, 1 \mathrm{mM} \mathrm{UDM}, 30 \mathrm{mM}$ imidazole] in a gravity flow column and eluted with $20 \mathrm{ml}$ of elution buffer [300 mM imidazole, $30 \mathrm{mM}$ phosphate buffer ( $\mathrm{pH}$ 7), $120 \mathrm{mM} \mathrm{NaCl}, 1 \mathrm{mM}$ UDM, 5\% glycerol]. The protein was concentrated to $0.6-0.8 \mathrm{mg} / \mathrm{ml}$ prior to size exclusion chromatography (SEC). SEC was done with the purified protein using Superdex 200 increase column preequilibrated with $30 \mathrm{mM}$ phosphate buffer (pH 7), $120 \mathrm{mM} \mathrm{NaCl}, 1 \mathrm{mM}$ UDM and 5\% glycerol.

\section{Determination of oligomeric status of QacA}

SEC-Multi-Angle Light Scattering was done using a three-detector system with light scattering detector, refractive index detector and UV detector to determine the molecular weight of QacA. The molecular weight of the protein was determined using protein conjugate mass analysis [54] with $\mathrm{dn} / \mathrm{dc}=0.18$ for globular protein and $\mathrm{dn} / \mathrm{dc}=0.1506$ for UDM. SEC- Multi-Angle Light Scattering [in $30 \mathrm{mM}$ phosphate buffer (pH 7.0), $120 \mathrm{mM}$ $\mathrm{NaCl}, 1 \mathrm{mM}$ UDM and 5\% glycerol (v/v)] was done with purified protein using Superdex 200 increase column.

\section{ATPase purification}

$\mathrm{F}_{0} \mathrm{~F}_{1}$ ATPase was expressed in E.coli DK8 cells and the membrane was purified using 50 $\mathrm{mM}$ Hepes and $100 \mathrm{mM} \mathrm{NaCl}$ following standard protocol. The membrane was homogenized in extraction buffer [50 mM Hepes buffer (pH 7.5), $100 \mathrm{mM} \mathrm{KCl}, 250 \mathrm{mM}$ sucrose, $40 \mathrm{mM} \varepsilon$-aminocaproic acid, $5 \mathrm{mM} \mathrm{MgSO}_{4}, 0.6 \%$ soybean lipid, $1.5 \%$ octyl glucoside, $0.6 \%$ sodium cholate, $0.6 \%$ sodium deoxycholate, $2.5 \%$ glycerol) and incubated for 1 hour. The solubilized membranes were centrifuged at 130,000 $\mathrm{rcf}$ for $1 \mathrm{~h}$. Ni-NTA resin (pre-equilibrated in extraction buffer) was used for binding by nutating with it for $1 \mathrm{~h}$ at $4{ }^{\circ} \mathrm{C}$. The resin bound to protein was washed $(25 \mathrm{mM}$ imidazole, extraction buffer) in a gravity flow column and eluted with $10 \mathrm{ml}$ of elution buffer $(250 \mathrm{mM}$ imidazole, extraction Buffer). SDS-PAGE was run post purification to check for the presence for all the subunits. 


\section{Reconstitution of WT QacA in proteoliposomes}

POPE and POPG (3:1) pure lipids (Avanti) were mixed with MMK buffer [10 mM MOPS (pH-6.5), $100 \mathrm{mM} \mathrm{KCl}, 5 \mathrm{mM} \mathrm{MgCl} 2$ ]. The final concentration was kept at $10 \mathrm{mg} / \mathrm{ml}$. Lipids were vortexed until completely homogenized with no visible clumps. Lipids were flash frozen in liquid nitrogen and thawed in warm water for eight cycles. Liposomes were then extruded using $200 \mathrm{~nm}$ polycarbonate filters (Whatman) for 21 cycles. For reconstitution $250 \mu \mathrm{l}$ liposomes were destabilized by $0.65 \%$ (final concentration) of sodium cholate. QacA and ATPase (2:1 molar ratio) were incubated with the liposomes for $30 \mathrm{~min}$ at RT; equal amount of ATPase was incubated with liposomes as a ATPase control. Detergent was removed using sephadex G25 beads (Sigma) and the sample was collected in a final volume of $1.5 \mathrm{ml}$. For assay, liposomes $(100 \mu \mathrm{l})$ were added in $2 \mathrm{ml}$ of MMK buffer (pH 6.5) containing $130 \mathrm{nM}$ valinomycin and $200 \mathrm{nM}$ ACMA $\left(\lambda_{\mathrm{Ex}}=410 \mathrm{~nm}, \lambda_{\mathrm{Em}}=480 \mathrm{~nm}\right)$, ATP $(130 \mu \mathrm{M})$ was added at $50^{\text {th }}$ second, substrate (TPP $0.5 \mathrm{mM}$, Pm $250 \mu \mathrm{M}$, Dq $1 \mathrm{mM}$ ) was added at $250^{\text {th }}$ second, $\mathrm{NH}_{4} \mathrm{Cl}(4 \mathrm{mM})$ at $300^{\text {th }}$ second was added as a uncoupler to abolish the $\mathrm{pH}$ gradient.

\section{Binding assay}

Binding assay was done using microscale thermophoresis (Nanotemper) [22]. Final protein concentration used for the assay was $50 \mathrm{nM}$. The protein was labeled with $50 \mathrm{nM}$ red TrisNTA dye in the C-terminus of the His-tag. Monolith ${ }^{\mathrm{TM}}$ NT.115 MST premium coated capillaries were used in each experiment. In the binding reaction, the protein concentration was kept constant. The protein was incubated with 16 two-fold serial dilutions of the ligand. The ligand was solubilized in protein containing buffer $[30 \mathrm{mM}$ phosphate buffer, $(\mathrm{pH} 7.0)$, $120 \mathrm{mM} \mathrm{NaCl}, 1 \mathrm{mM}$ UDM, 5\% glycerol]. The starting concentrations of the ligands were $10 \mathrm{mM}$ in case of TPP and Dq, and $5 \mathrm{mM}$ of Pm. The experiments were done with two independent sets of purified protein.

\section{pH dependence of fluorescein's fluorescence}

Fluorescein is a $\mathrm{pH}$ sensitive dye which fluoresces greater as the $\mathrm{pH}$ increases. For substrateinduced proton release assay done for this study, a standard curve of fluorescence at different $\mathrm{pH}$ was plotted with $2 \mu \mathrm{M}$ of fluorescein in Fluoromax-3 (Horiba) fluorescence spectrophotometer with an integration time of $1 \mathrm{~s}$ at $\lambda_{\mathrm{Ex}}=494 \mathrm{~nm}$ and $\lambda_{\mathrm{Em}}=521 \mathrm{~nm}$. Acetate buffer (10 mM) was used for a $\mathrm{pH}$ range of 2-6, $10 \mathrm{mM}$ Hepes was used for $\mathrm{pH}$ 7-8 and $10 \mathrm{mM}$ bicine was used for $\mathrm{pH} 9-10$. Experiment was repeated three times.

\section{Substrate-induced proton release assay}

The assay was done with purified QacA $(6 \mu \mathrm{M})$. Purified protein was dialyzed with unbuffered solution ( $1 \mathrm{~L} ; 200 \mathrm{mM} \mathrm{NaCl}, 0.8 \mathrm{mM} \mathrm{UDM})$ to remove buffering agents. Fluorescein, a pH sensitive dye was used to detect the change in $\mathrm{H}^{+}$concentration of the solution during the assay. Fluorescein $(2 \mu \mathrm{M})$ was added at the start; at $50^{\text {th }}$ second $6 \mu \mathrm{M}$ $\mathrm{HCl}$ and at $100^{\text {th }}, 150^{\text {th }}$ and $200^{\text {th }}$ second $200 \mu \mathrm{M}$ of the substrate (TPP, Pm and Dq) were added in the respective experiments. Control experiment was done in blank $(200 \mathrm{mM} \mathrm{NaCl}$, $0.8 \mathrm{mM}$ UDM) solution following the same procedure $[38,55]$. Total duration of the experiment was 250 seconds. Fluorescein's fluorescence was measured at $\lambda_{\mathrm{Ex}}=494 \mathrm{~nm}$, 
$\lambda_{\mathrm{Em}}=521 \mathrm{~nm}$ in Fluoromax-3 (Horiba) fluorescence spectrophotometer with integration time of $1 \mathrm{~s}$. The experiment was performed with a single batch of protein.

\section{Supplementary Material}

Refer to Web version on PubMed Central for supplementary material.

\section{Acknowledgements}

The authors would like to thank Prof. B. Gopal, Dr. Vinothkumar Kutti Ragunath and Dr. Abhijit A. Sardesai for critical reading of the manuscript. Authors are grateful to Ms. Swati Dubey, CDFD and Dr. Abhijit A. Sardesai, CDFD for the gift of JD838 strain. The authors thank Dr. David Drew, University of Stockholm for the gift of the $\mathrm{F}_{0} \mathrm{~F}_{1}$ ATPase clone and purification protocol. PM is supported by the IISc-GATE PhD fellowship. AA is a student of the IISc-Integrated PhD program. NH is support by DBT-JRF PhD fellowship. This work was supported by the Wellcome Trust/DBT India Alliance Fellowship [IA/1/15/2/502063] awarded to AP. AP is a recipient of the Innovative Young Biotechnologist Award (IYBA) [BT/09/IYBA/2015/13] from the Dept. of Biotechnology (DBT), India. The authors acknowledge the DBT-IISc partnership program phase-I and phase-II support to carry out this work

\section{Abbreviations}

ACMA

TM

DHA

Et

TPP

Dq

Pm

$\mathbf{C x}$

POT

MATE

$\lambda_{\mathbf{E x}} / \lambda_{\mathbf{E m}}$

MST

EL

FSEC

UDM

PEG-Mal

WT

$\mathbf{E}$
9-Amino-6-Chloro-2-Methoxyacridine

Transmembrane

Drug-Proton Antiporter/Drug- $\mathrm{H}^{+}$Antiporter

Ethidium

Tetraphenylphosphonium

Dequalinium

Pentamidine

Chlorhexidine

Proton-dependent Oligopeptide Transporter

multidrug and toxin extrusion

Excitation/Emission Wavelength

Microscale Thermophoresis

Extracellular Loop

Fluorescence-detection Size Exclusion Chromatography

Undecyl- $\beta$-D-Maltopyranoside

Polyethylene Glycol-Maleimide

Wild type

Empty vector 


\section{References}

[1]. Piddock LJ. Multidrug-resistance efflux pumps - not just for resistance. Nat Rev Microbiol. 2006; 4:629-36. [PubMed: 16845433]

[2]. Du D, Wang-Kan X, Neuberger A, van Veen HW, Pos KM, Piddock LJV, et al. Multidrug efflux pumps: structure, function and regulation. Nat Rev Microbiol. 2018

[3]. Chitsaz M, Brown MH. The role played by drug efflux pumps in bacterial multidrug resistance. Essays Biochem. 2017; 61:127-39. [PubMed: 28258236]

[4]. Costa SS, Viveiros M, Amaral L, Couto I. Multidrug Efflux Pumps in Staphylococcus aureus: an Update. Open Microbiol J. 2013; 7:59-71. [PubMed: 23569469]

[5]. Schindler BD, Kaatz GW. Multidrug efflux pumps of Gram-positive bacteria. Drug Resist Updat. 2016; 27:1-13. [PubMed: 27449594]

[6]. Reddy VS, Shlykov MA, Castillo R, Sun EI, Saier MH Jr. The major facilitator superfamily (MFS) revisited. FEBS J. 2012; 279:2022-35. [PubMed: 22458847]

[7]. Heng J, Zhao Y, Liu M, Liu Y, Fan J, Wang X, et al. Substrate-bound structure of the E. coli multidrug resistance transporter MdfA. Cell Res. 2015; 25:1060-73. [PubMed: 26238402]

[8]. Yin Y, He X, Szewczyk P, Nguyen T, Chang G. Structure of the multidrug transporter EmrD from Escherichia coli. Science. 2006; 312:741-4. [PubMed: 16675700]

[9]. Jiang D, Zhao Y, Wang X, Fan J, Heng J, Liu X, et al. Structure of the YajR transporter suggests a transport mechanism based on the conserved motif A. Proc Natl Acad Sci U S A. 2013; 110:14664-9. [PubMed: 23950222]

[10]. Adler J, Bibi E. Promiscuity in the geometry of electrostatic interactions between the Escherichia coli multidrug resistance transporter MdfA and cationic substrates. J Biol Chem. 2005; 280:2721-9. [PubMed: 15557318]

[11]. Adler J, Bibi E. Determinants of substrate recognition by the Escherichia coli multidrug transporter MdfA identified on both sides of the membrane. J Biol Chem. 2004; 279:8957-65. [PubMed: 14688269]

[12]. Schaedler TA, van Veen HW. A flexible cation binding site in the multidrug major facilitator superfamily transporter LmrP is associated with variable proton coupling. FASEB J. 2010; 24:3653-61. [PubMed: 20472749]

[13]. Linial M. Vesicular transporters join the major facilitator superfamily (MFS). Trends Biochem Sci. 1993; 18:248-9. [PubMed: 8212132]

[14]. Chaudhry FA, Boulland JL, Jenstad M, Bredahl MK, Edwards RH. Pharmacology of neurotransmitter transport into secretory vesicles. Handb Exp Pharmacol. 2008:77-106. [PubMed: 18064412]

[15]. Edgar R, Bibi E. A single membrane-embedded negative charge is critical for recognizing positively charged drugs by the Escherichia coli multidrug resistance protein MdfA. EMBO J. 1999; 18:822-32. [PubMed: 10022825]

[16]. Yaffe D, Vergara-Jaque A, Shuster Y, Listov D, Meena S, Singh SK, et al. Functionally important carboxyls in a bacterial homologue of the vesicular monoamine transporter (VMAT). J Biol Chem. 2014; 289:34229-40. [PubMed: 25336661]

[17]. Paulsen IT, Brown MH, Littlejohn TG, Mitchell BA, Skurray RA. Multidrug resistance proteins QacA and QacB from Staphylococcus aureus: membrane topology and identification of residues involved in substrate specificity. Proc Natl Acad Sci U S A. 1996; 93:3630-5. [PubMed: 8622987]

[18]. Drew D, Boudker O. Shared Molecular Mechanisms of Membrane Transporters. Annu Rev Biochem. 2016; 85:543-72. [PubMed: 27023848]

[19]. Solcan N, Kwok J, Fowler PW, Cameron AD, Drew D, Iwata S, et al. Alternating access mechanism in the POT family of oligopeptide transporters. EMBO J. 2012; 31:3411-21. [PubMed: 22659829]

[20]. Newstead S. Molecular insights into proton coupled peptide transport in the PTR family of oligopeptide transporters. Biochim Biophys Acta. 2015; 1850:488-99. [PubMed: 24859687] 
[21]. Alegre KO, Paul S, Labarbuta P, Law CJ. Insight into determinants of substrate binding and transport in a multidrug efflux protein. Sci Rep. 2016; 6:22833. [PubMed: 26961153]

[22]. Martinez Molledo M, Quistgaard EM, Flayhan A, Pieprzyk J, Low C. Multispecific Substrate Recognition in a Proton-Dependent Oligopeptide Transporter. Structure. 2018; 26:467-76 e4. [PubMed: 29429879]

[23]. Quistgaard EM, Low C, Guettou F, Nordlund P. Understanding transport by the major facilitator superfamily (MFS): structures pave the way. Nat Rev Mol Cell Biol. 2016; 17:123-32. [PubMed: 26758938]

[24]. Mitchell BA, Paulsen IT, Brown MH, Skurray RA. Bioenergetics of the staphylococcal multidrug export protein QacA. Identification of distinct binding sites for monovalent and divalent cations. J Biol Chem. 1999; 274:3541-8. [PubMed: 9920900]

[25]. Mitchell BA, Brown MH, Skurray RA. QacA multidrug efflux pump from Staphylococcus aureus: comparative analysis of resistance to diamidines, biguanidines, and guanylhydrazones. Antimicrob Agents Chemother. 1998; 42:475-7. [PubMed: 9527814]

[26]. Blakely RD, Edwards RH. Vesicular and plasma membrane transporters for neurotransmitters. Cold Spring Harb Perspect Biol. 2012; 4

[27]. Schuldiner S. Competition as a way of life for H(+)-coupled antiporters. J Mol Biol. 2014; 426:2539-46. [PubMed: 24862284]

[28]. Yaffe D, Forrest LR, Schuldiner S. The ins and outs of vesicular monoamine transporters. J Gen Physiol. 2018; 150:671-82. [PubMed: 29666153]

[29]. Zhang XC, Liu M, Lu G, Heng J. Thermodynamic secrets of multidrug resistance: A new take on transport mechanisms of secondary active antiporters. Protein Sci. 2018; 27:595-613. [PubMed: 29193407]

[30]. Fowler PW, Orwick-Rydmark M, Radestock S, Solcan N, Dijkman PM, Lyons JA, et al. Gating topology of the proton-coupled oligopeptide symporters. Structure. 2015; 23:290-301. [PubMed: 25651061]

[31]. Roy A, Kucukural A, Zhang Y. I-TASSER: a unified platform for automated protein structure and function prediction. Nat Protoc. 2010; 5:725-38. [PubMed: 20360767]

[32]. Wang Y, Toei M, Forgac M. Analysis of the membrane topology of transmembrane segments in the C-terminal hydrophobic domain of the yeast vacuolar ATPase subunit a (Vph1p) by chemical modification. J Biol Chem. 2008; 283:20696-702. [PubMed: 18508769]

[33]. Jin J, Guffanti AA, Beck C, Krulwich TA. Twelve-transmembrane-segment (TMS) version (DeltaTMS VII-VIII) of the 14-TMS Tet(L) antibiotic resistance protein retains monovalent cation transport modes but lacks tetracycline efflux capacity. J Bacteriol. 2001; 183:2667-71. [PubMed: 11274128]

[34]. Hassan KA, Xu Z, Watkins RE, Brennan RG, Skurray RA, Brown MH. Optimized production and analysis of the staphylococcal multidrug efflux protein QacA. Protein Expr Purif. 2009; 64:118-24. [PubMed: 19073264]

[35]. Lewinson O, Bibi E. Evidence for simultaneous binding of dissimilar substrates by the Escherichia coli multidrug transporter MdfA. Biochemistry. 2001; 40:12612-8. [PubMed: 11601985]

[36]. Masureel M, Martens C, Stein RA, Mishra S, Ruysschaert JM, McHaourab HS, et al. Protonation drives the conformational switch in the multidrug transporter LmrP. Nat Chem Biol. 2014; 10:149-55. [PubMed: 24316739]

[37]. Bolhuis H, van Veen HW, Brands JR, Putman M, Poolman B, Driessen AJ, et al. Energetics and mechanism of drug transport mediated by the lactococcal multidrug transporter LmrP. J Biol Chem. 1996; 271:24123-8. [PubMed: 8798651]

[38]. Fluman N, Adler J, Rotenberg SA, Brown MH, Bibi E. Export of a single drug molecule in two transport cycles by a multidrug efflux pump. Nat Commun. 2014; 5:4615. [PubMed: 25105370]

[39]. Deng D, Xu C, Sun P, Wu J, Yan C, Hu M, et al. Crystal structure of the human glucose transporter GLUT1. Nature. 2014; 510:121-5. [PubMed: 24847886]

[40]. Vullo S, Bonifacio G, Roy S, Johner N, Berneche S, Kellenberger S. Conformational dynamics and role of the acidic pocket in ASIC pH-dependent gating. Proc Natl Acad Sci U S A. 2017; 114:3768-73. [PubMed: 28320963] 
[41]. Yan N. Structural Biology of the Major Facilitator Superfamily Transporters. Annu Rev Biophys. 2015; 44:257-83. [PubMed: 26098515]

[42]. Merickel A, Rosandich P, Peter D, Edwards RH. Identification of residues involved in substrate recognition by a vesicular monoamine transporter. J Biol Chem. 1995; 270:25798-804. [PubMed: 7592763]

[43]. Tirosh O, Sigal N, Gelman A, Sahar N, Fluman N, Siemion S, et al. Manipulating the drug/proton antiport stoichiometry of the secondary multidrug transporter MdfA. Proc Natl Acad Sci U S A. 2012; 109:12473-8. [PubMed: 22802625]

[44]. Bolhuis H, van Veen HW, Poolman B, Driessen AJ, Konings WN. Mechanisms of multidrug transporters. FEMS Microbiol Rev. 1997; 21:55-84. [PubMed: 9299702]

[45]. Henderson RK, Fendler K, Poolman B. Coupling efficiency of secondary active transporters. Curr Opin Biotechnol. 2018; 58:62-71. [PubMed: 30502621]

[46]. Lu M, Radchenko M, Symersky J, Nie R, Guo Y. Structural insights into H+-coupled multidrug extrusion by a MATE transporter. Nat Struct Mol Biol. 2013; 20:1310-7. [PubMed: 24141706]

[47]. Panahi A, Brooks CL 3rd. Membrane environment modulates the pKa values of transmembrane helices. J Phys Chem B. 2015; 119:4601-7. [PubMed: 25734901]

[48]. Henderson R, Poolman B. Proton-solute coupling mechanism of the maltose transporter from Saccharomyces cerevisiae. Sci Rep. 2017; 7:14375. [PubMed: 29084970]

[49]. Otsuka M, Yasuda M, Morita Y, Otsuka C, Tsuchiya T, Omote H, et al. Identification of essential amino acid residues of the NorM Na+/multidrug antiporter in Vibrio parahaemolyticus. $\mathbf{J}$ Bacteriol. 2005; 187:1552-8. [PubMed: 15716425]

[50]. Moriyama Y, Iwamoto A, Hanada H, Maeda M, Futai M. One-step purification of Escherichia coli $\mathrm{H}(+)$ - ATPase (F0F1) and its reconstitution into liposomes with neurotransmitter transporters. J Biol Chem. 1991; 266:22141-6. [PubMed: 1834667]

[51]. Ebejer JP, Hill JR, Kelm S, Shi J, Deane CM. Memoir: template-based structure prediction for membrane proteins. Nucleic Acids Res. 2013; 41:W379-83. [PubMed: 23640332]

[52]. Huang J, Rauscher S, Nawrocki G, Ran T, Feig M, de Groot BL, et al. CHARMM36m: an improved force field for folded and intrinsically disordered proteins. Nat Methods. 2017; 14:713. [PubMed: 27819658]

[53]. Van Der Spoel D, Lindahl E, Hess B, Groenhof G, Mark AE, Berendsen HJ. GROMACS: fast, flexible, and free. J Comput Chem. 2005; 26:1701-18. [PubMed: 16211538]

[54]. Slotboom DJ, Duurkens RH, Olieman K, Erkens GB. Static light scattering to characterize membrane proteins in detergent solution. Methods. 2008; 46:73-82. [PubMed: 18625320]

[55]. Soskine M, Adam Y, Schuldiner S. Direct evidence for substrate-induced proton release in detergent-solubilized EmrE, a multidrug transporter. J Biol Chem. 2004; 279:9951-5. [PubMed: 14701800] 


\section{Highlights}

- A homology model of QacA with 14 TM helices was built and used to test the discrete roles of acidic residues within the vestibule in substrate recognition and $\mathrm{H}^{+}$-coupled antibacterial efflux.

- Four out of six residues identified in the binding pocket play essential or conditional roles in the transport of diverse substrates.

- $\quad$ Acidic residues in the cytosolic half of TM13 (E407, D411) are important for promiscuous substrate recognition and enhancing the $\mathrm{H}^{+}$:drug stoichiometry during translocation. 
(a)

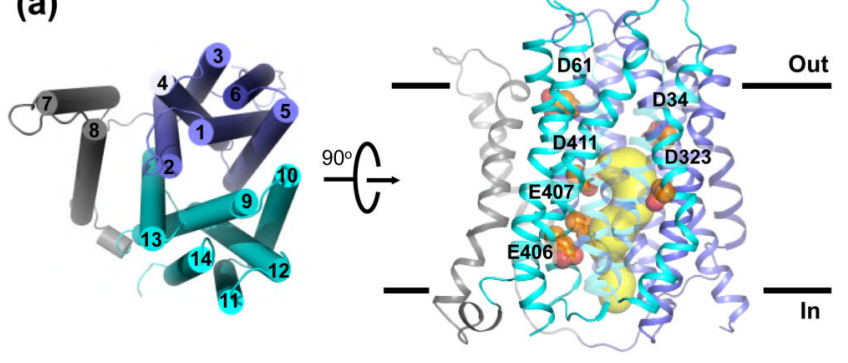

(b)

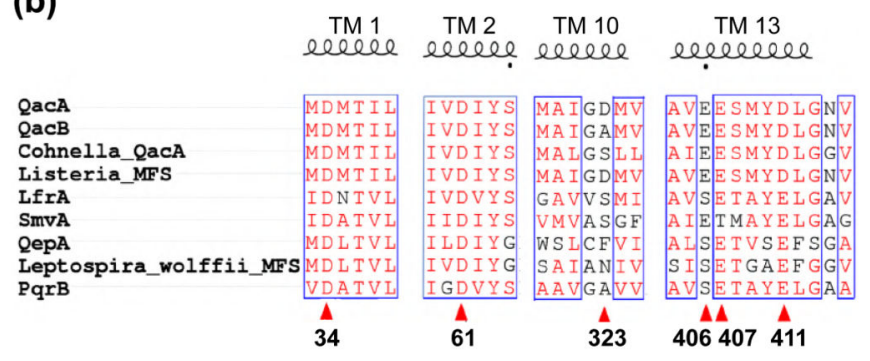

(c)

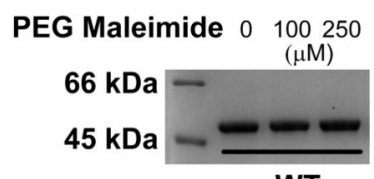

WT

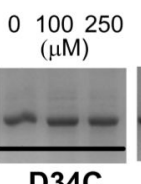

D34C

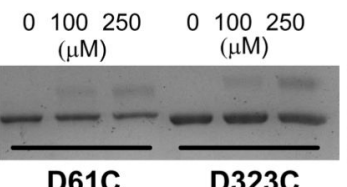

D61C

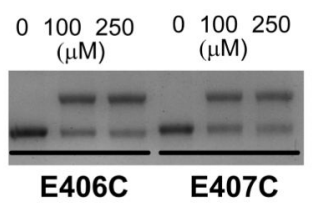

0100250 $(\mu \mathrm{M})$

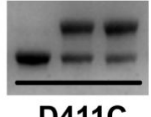

Figure 1.

Homology model of QacA. (a) Model shows TMs 1-6 (dark blue) and TMs 9-14 (cyan) displaying pseudo two-fold symmetry. TMs 7 and 8 (grey) connect the two domains. Acidic residues in the vestibule are indicated in orange. The model displays an inward-open conformation with solvent accessible vestibule colored in yellow. (b) Multiple sequence alignment of QacA and its homologs across various species. Acidic residues mapped in QacA vestibule are pointed with red arrows. (c) PEG-maleimide (5 kDa) accessibility assay indicates band shifts to higher molecular mass due to PEG-maleimide covalently modifying individual cysteine mutants. 

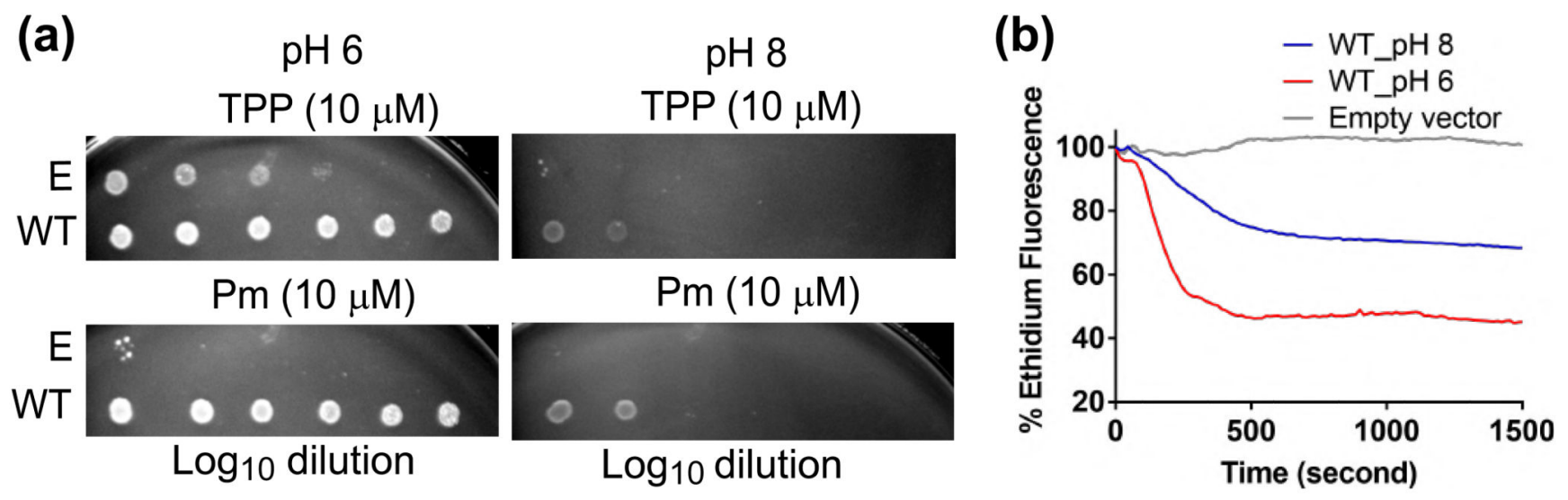

Figure 2.

$\Delta \mathrm{pH}$ dependence of QacA's activity checked by (a) survival and (WT-Wild Type QacA, EEmpty vector) (b) whole cell (ethidium, $\lambda_{\mathrm{Ex}}=530 \mathrm{~nm}, \lambda_{\mathrm{Em}}=610 \mathrm{~nm}$ ) efflux assays done at external $\mathrm{pH}$ of 6 and 8 . All assays were independently performed at least twice. 

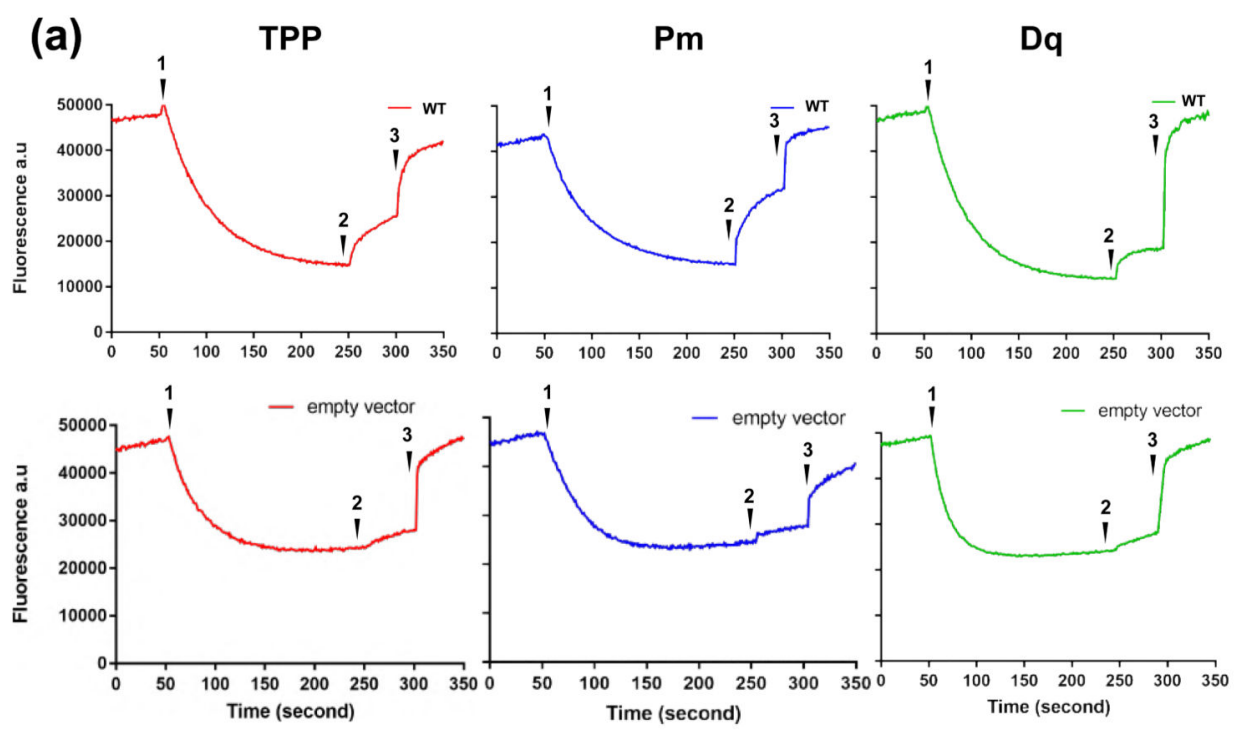

(b)
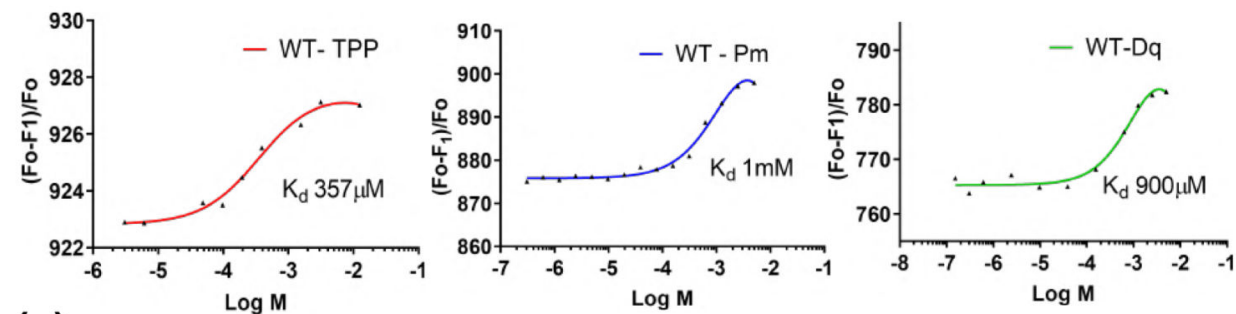

(c)
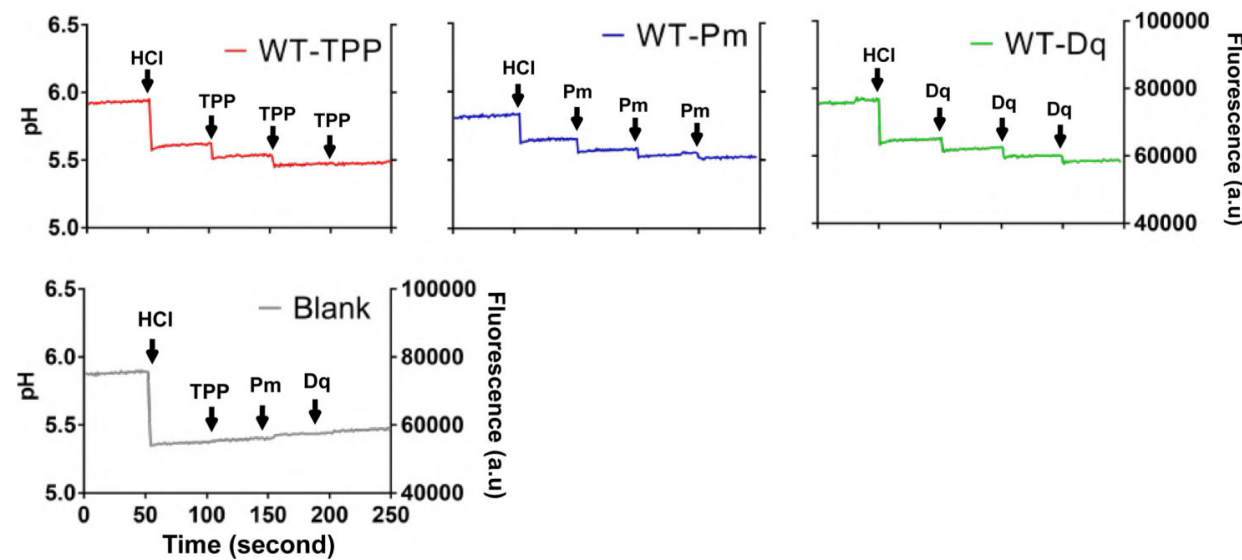

Figure 3.

Functional characterization of WT QacA. (a) substrate-induced $\mathrm{H}^{+}$transport assay in insideout vesicles. WT QacA is transport active for all the drugs tested $(n=2)$. Addition of ATP at $50^{\text {th }}$ second is denoted by $\mathbf{1} ; \mathbf{2}$ represents substrate addition at $250^{\text {th }}$ second and $\mathbf{3}$ indicates addition of uncoupler to abolish the $\mathrm{H}^{+}$gradient at $300^{\text {th }}$ second. The experiment was done in presence of $\mathrm{pH}$ gradient sensitive dye ACMA ( $\left.\lambda_{\mathrm{Ex}}=409 \mathrm{~nm}, \lambda_{\mathrm{Em}}=474 \mathrm{~nm}\right)$. (b) The $K_{\mathrm{d}}$ estimated from the binding assays done using microscale thermophoresis displays submillimolar affinity for TPP, Pm and Dq $(n=2)$. (c) Fluorescein's fluorescence $\left(\lambda_{\mathrm{Ex}}=494 \mathrm{~nm}\right.$, 
$\lambda_{E m}=521 \mathrm{~nm}$ ) quenching experiment indicates substrate-induced $\mathrm{H}^{+}$release. The assay was done with a single batch of protein. 
(a)

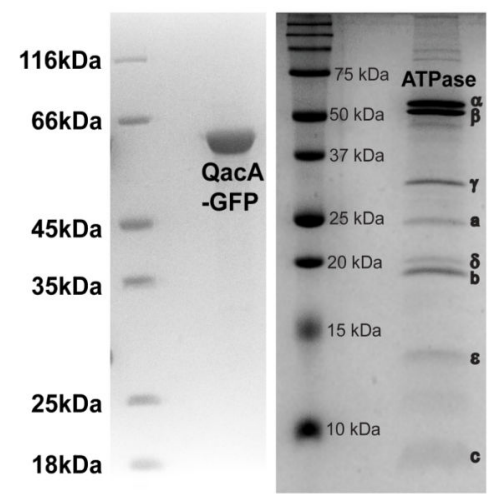

(b)

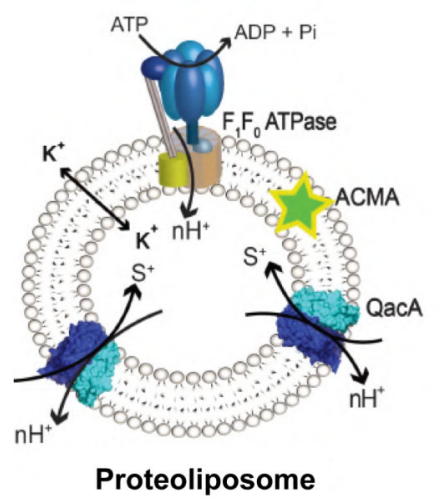

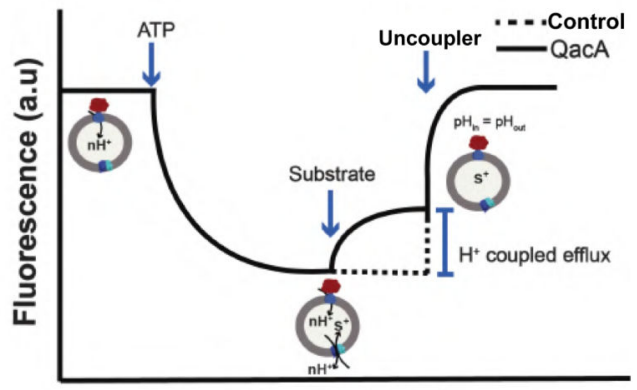

Time (second)

(c)
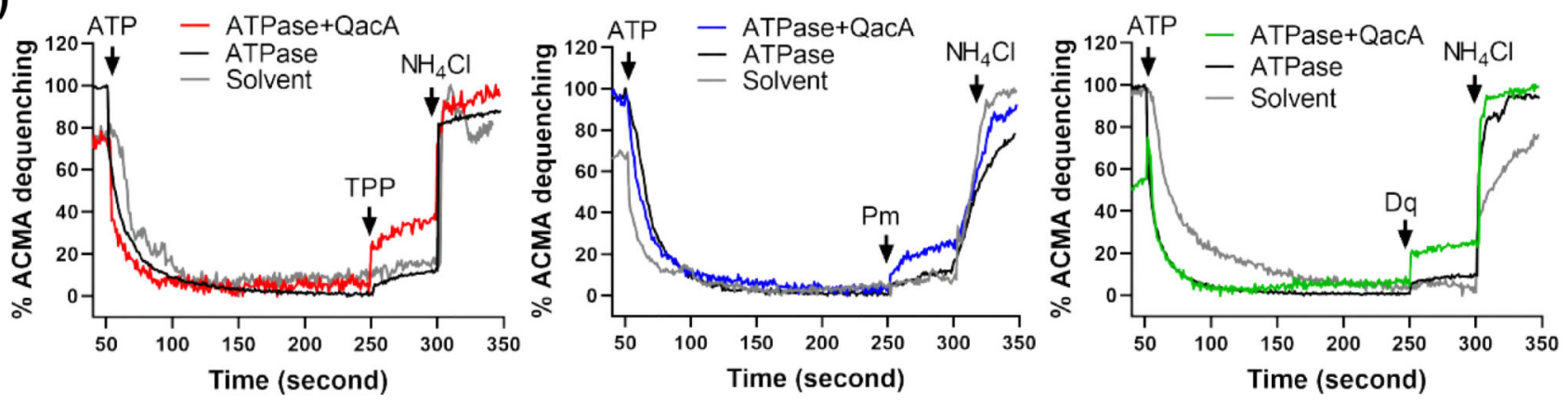

Figure 4.

Substrate-induced $\mathrm{H}^{+}$transport activity of QacA assayed using proteoliposomes (POPE:POPG (3:1)) reconstituted with purified QacA-GFP and E.coli $\mathrm{F}_{0} \mathrm{~F}_{1}$-ATPase. (a) NiNTA purified fractions of WT QacA-GFP and ATPase loaded on SDS-PAGE, (b) Schematic representation of WT QacA-GFP and $\mathrm{F}_{0} \mathrm{~F}_{1}$-ATPase reconstitution and substrate-induced $\mathrm{H}^{+}$ transport assay, (c) Reconstituted QacA displays substrate-induced $\mathrm{H}^{+}$transport in presence of TPP $(0.5 \mathrm{mM}), \operatorname{Pm}(0.25 \mathrm{mM})$ and $\mathrm{Dq}(1 \mathrm{mM})(\mathrm{n}=2)$, the experiment was done in presence of $\mathrm{pH}$ gradient sensitive dye $\operatorname{ACMA}\left(\lambda_{\mathrm{Ex}}=410 \mathrm{~nm}, \lambda_{\mathrm{Em}}=480 \mathrm{~nm}\right)$. The grey line in the panels indicate proteoliposomes with ATPase and QacA tested with the solvent instead of substrates and the black lines indicate proteoliposomes with only ATPase tested with all three substrates added at $250^{\text {th }}$ second. 
(a)

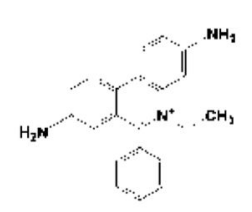

Ethidium<smiles>C1CCC(C2(C3CCCC3(C3CCCC3)C3CCCC3)CCCC2)C1</smiles>

TPP

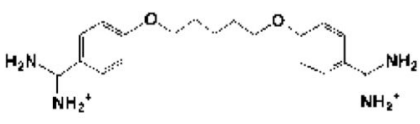

Pentamidine

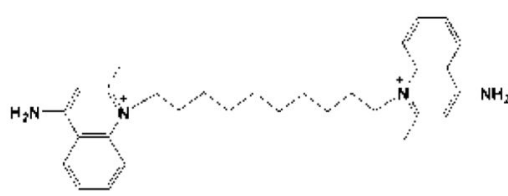

Dequalinium
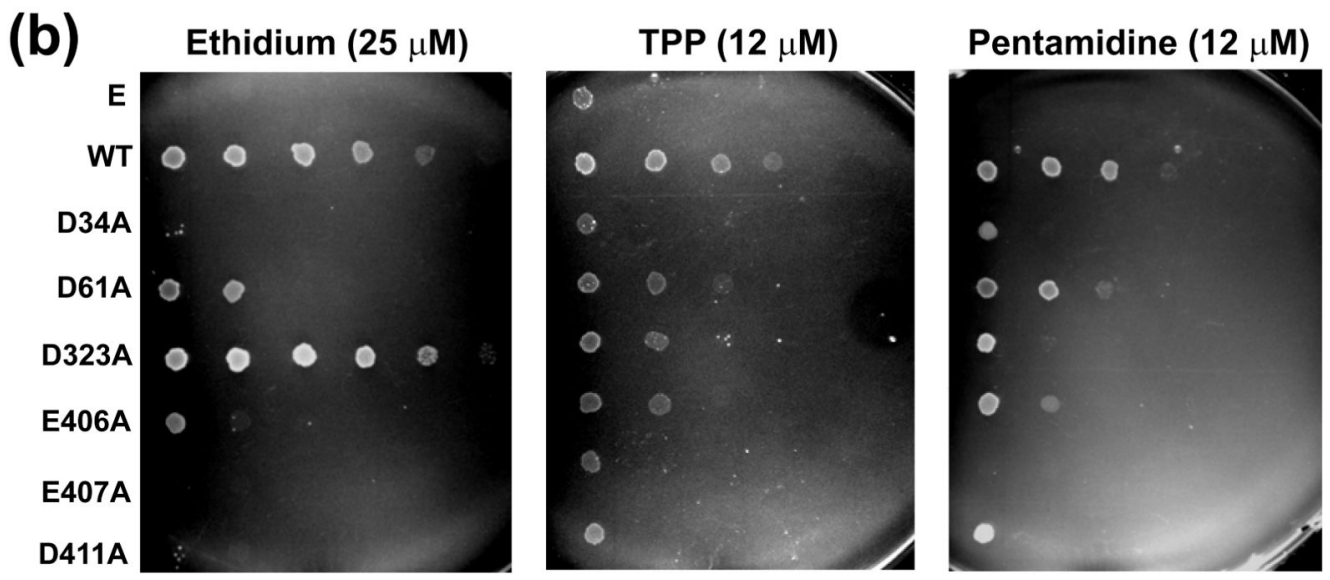

Dequalinium $(10 \mu \mathrm{M})$

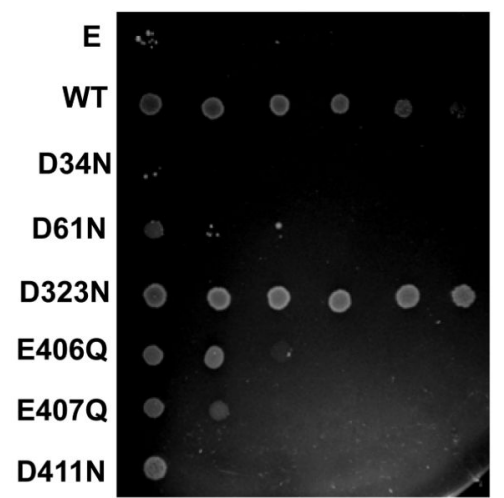

Log10 dilution

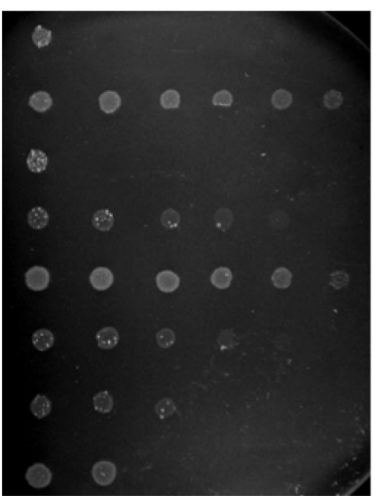

$\log _{10}$ dilution

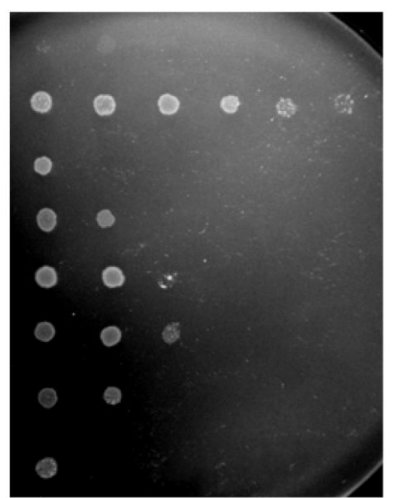

$\log _{10}$ dilution
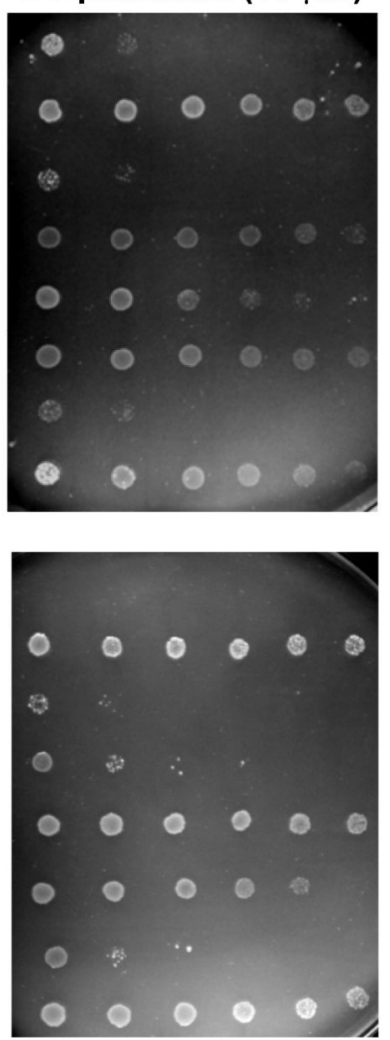

Log10 dilution

Figure 5.

Drug resistance in E.coli cells expressing QacA. (WT - Wild Type QacA, E - Empty Vector) (a) Chemical structures of the cationic dye (Et) and antibacterial compounds (TPP, Pm, Dq) used as substrates for QacA. (b) Survival assay in presence of both monovalent (Et, TPP) and divalent (Pm, Dq) cations identifies four acidic residues D34, D323, E407 and D411 as crucial for the survival of bacteria in presence of toxic substrates. The role of D61 and E406 seems to be comparatively less significant. 'E' represents empty vector containing negative control. $\log _{10}$ dilution starts with O.D. 1.0 at $\lambda=600 \mathrm{~nm}$ from left to right. Plates are incubated for 12 hours $(n=3)$. 
(a)

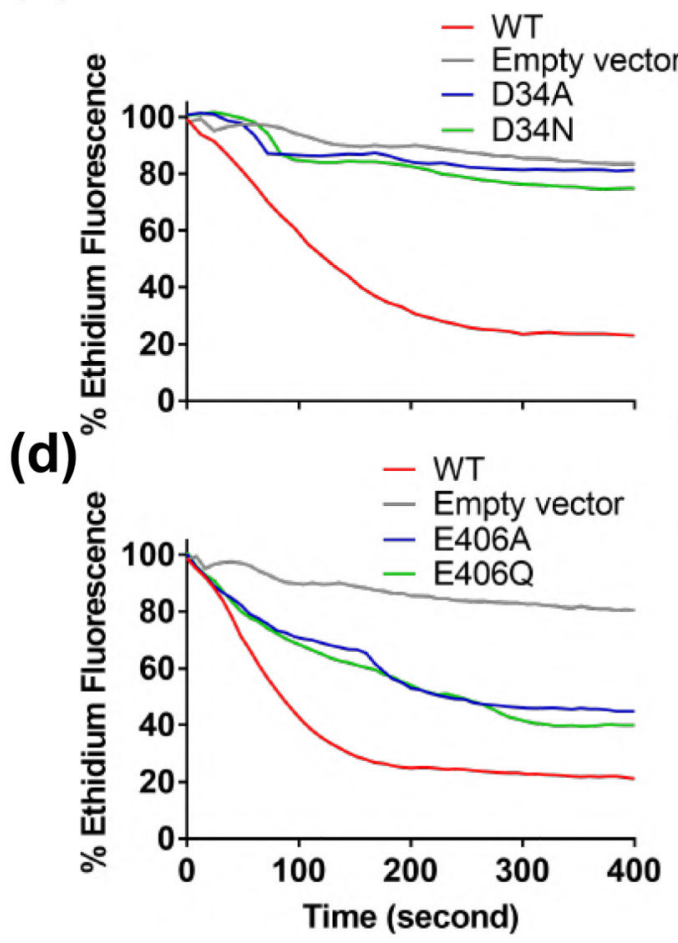

(b)

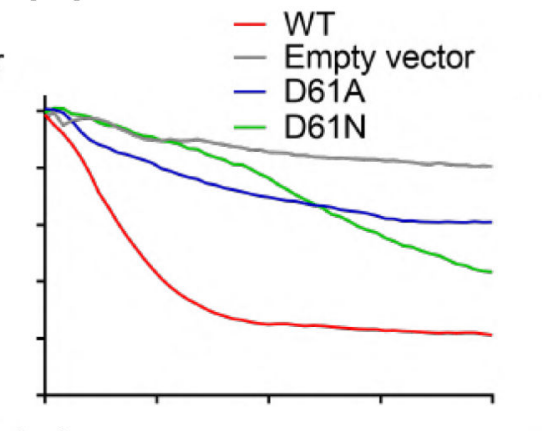

(e)

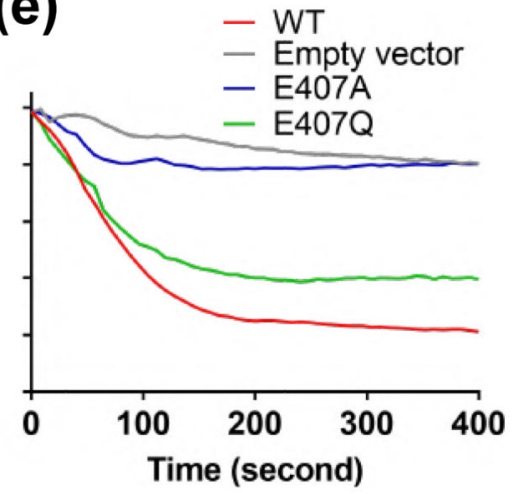

(c)
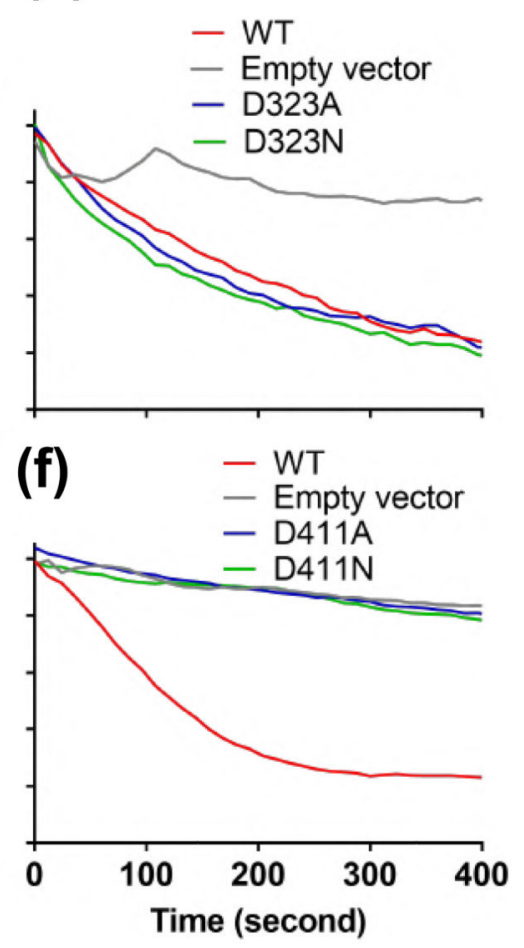

Figure 6.

Altered transport properties of QacA mutants. (a-f) Whole cell efflux assay with WT QacA and its single mutants expressed in JD838 cells incubated with $50 \mu \mathrm{M} \mathrm{EtBr}\left(\lambda_{\mathrm{Ex}}=530 \mathrm{~nm}\right.$, $\left.\lambda_{\mathrm{Em}}=610 \mathrm{~nm}\right)$ for 1 hour in presence of CCCP $(0.5 \mu \mathrm{M})(\mathrm{n}=3)$. Altered efflux was measured as a function of ethidium fluorescence loss over a duration of $400 \mathrm{~s}$. 
(a)

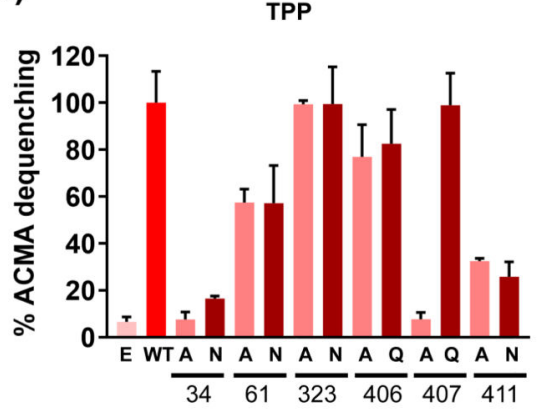

(b)

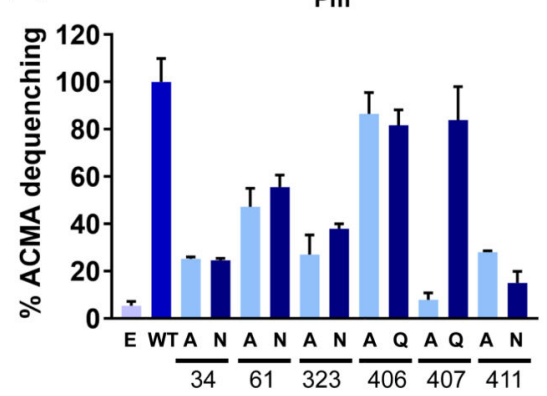

(c)

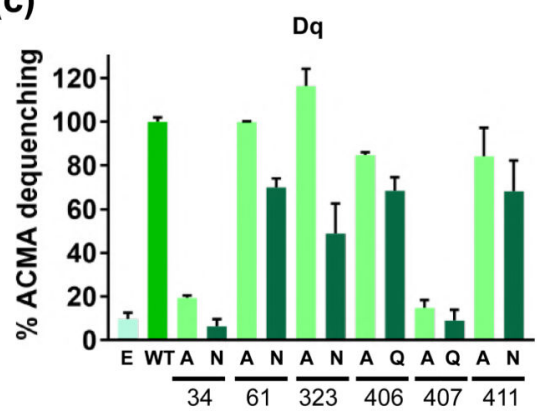

Figure 7.

Inside-out vesicle based TPP, Pm and Dq induced proton transport $(\mathrm{n}=2)$ assay (WT - Wild Type QacA, E - Empty vector). Valinomycin was kept throughout the experiment at $0.5 \mu \mathrm{M}$ to prevent the build-up of membrane potential. The formation of $\Delta \mathrm{pH}$ and the transport induced fluorescence dequenching of ACMA was measured $\left(\lambda_{\mathrm{Ex}}=409 \mathrm{~nm}, \lambda_{\mathrm{Em}}=474 \mathrm{~nm}\right)$. The extent of ACMA fluorescence dequenching, observed upon addition of the substrate to WT QacA vs. individual mutants is compared in the bar graph. Error bars represent the range observed for two independent measurements. 
(a)
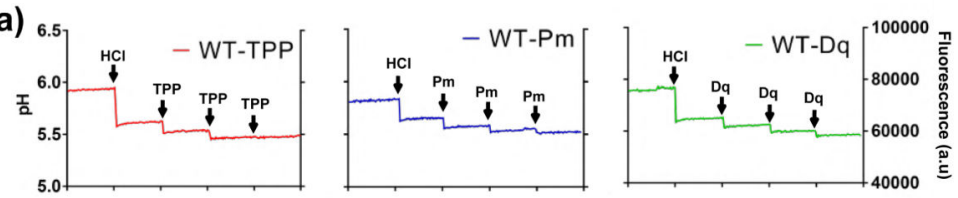

(b)
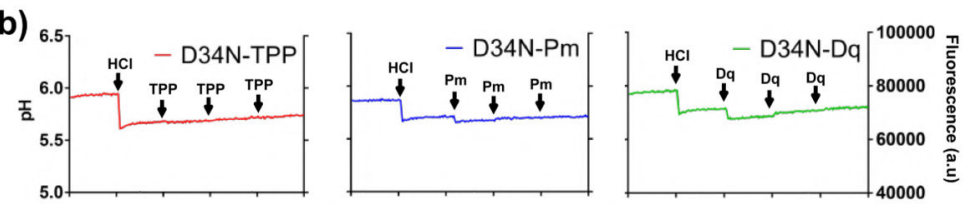

(c)
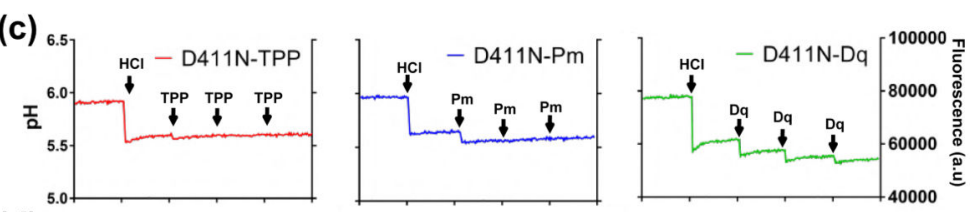

(d)
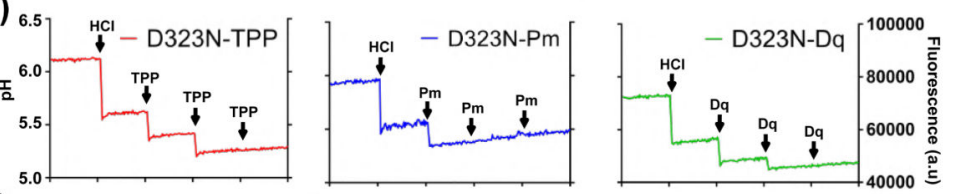

(e)
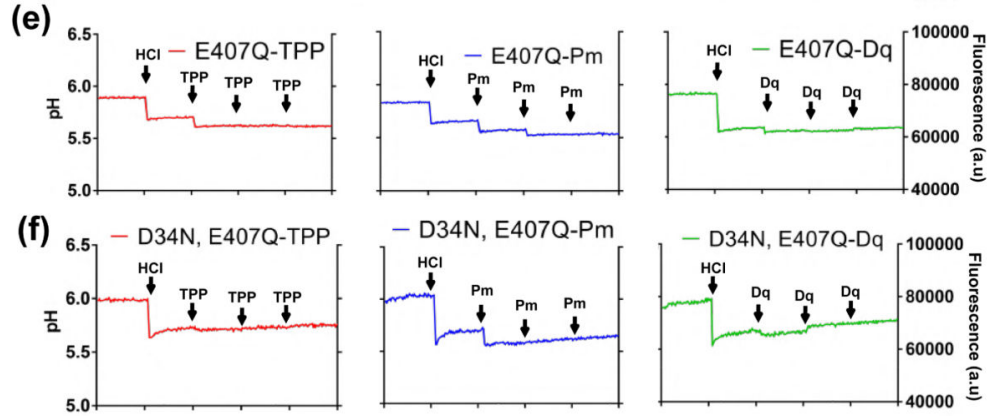

(g)
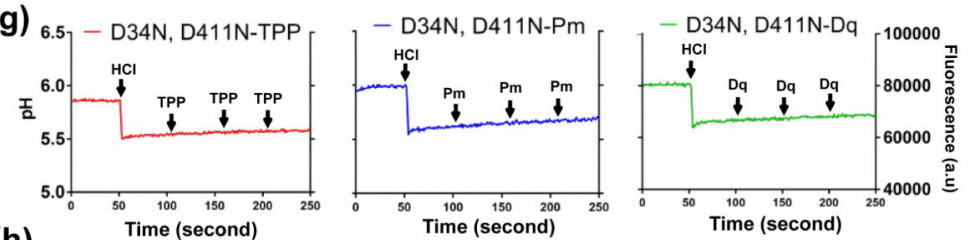

(h)

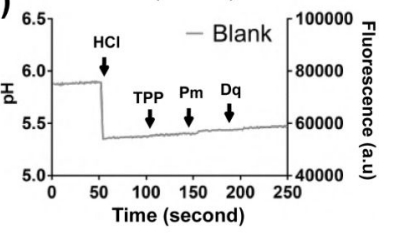

Figure 8.

Substrate-induced proton release. The assay was done with purified QacA (6 $\mu \mathrm{M}$, dialyzed to remove buffering agents). The $\mathrm{pH}$ of the solution was monitored in a time-dependent manner using fluorescein $(2 \mu \mathrm{M})$, a pH-sensitive dye $\left(\lambda_{\mathrm{Ex}}=494 \mathrm{~nm}, \lambda_{\mathrm{Em}}=521 \mathrm{~nm}\right)$. (a-g) Each row represents one mutant of QacA tested with three substrates. Arrows denote additions of $\mathrm{HCl}(6 \mu \mathrm{M})$ and titration of substrates $(200 \mu \mathrm{M})$. (h) The titration was repeated in unbuffered solution without protein, as a negative control. A single batch of pure protein was used for these measurements. 


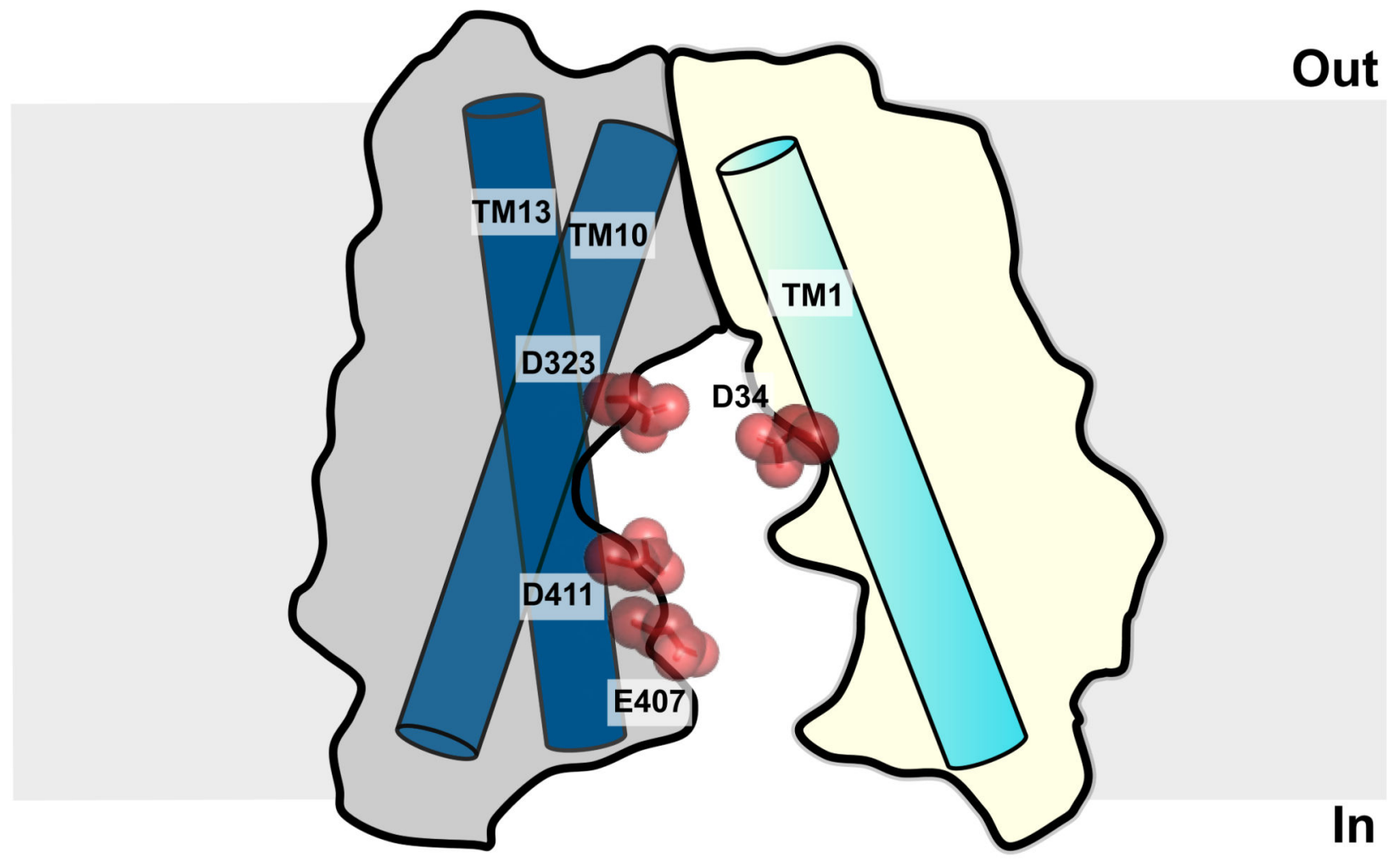

Out

Substrate Recognition site Protonation site

\section{D34, D411 E407}

TPP

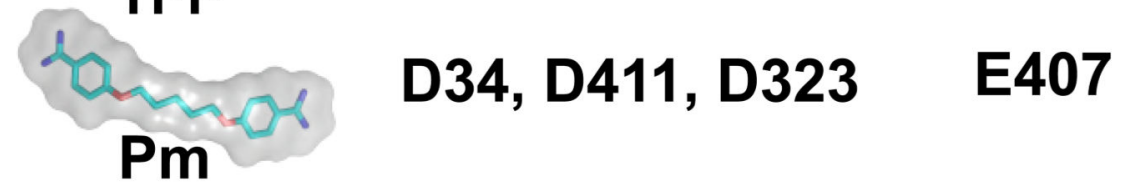

D34, E407

Dq

Figure 9.

Substrate recognition and promiscuity. Cartoon depicting the acidic residues present in the transport vestibule of QacA that are required for recognition and act as protonation sites helping in transport of the lipophilic cationic ligands. TPP transport requires D34, D411 and E407; Pm, a biguanide requires four residues for transport; Dq recognition and transport requires just two residues. The longer linker in Dq compared to Pm could result in the shift of the secondary recognition site from D411 to E407. 
Table 1

The dissociation constants $\left(K_{d}\right)$ are listed, analyzed from the binding experiments done using MST (microscale thermophoresis) carried out with TPP, Pm and Dq.

\begin{tabular}{|c|c|c|}
\hline mutants & ligand & $K_{\mathrm{d}}(\mathbf{m M})$ \\
\hline WT & TPP & $0.36 \pm 0.07$ \\
\hline D34N & TPP & - \\
\hline D61N & TPP & $0.93 \pm 0.10$ \\
\hline D323N & TPP & $0.30 \pm 0.05$ \\
\hline E406Q & TPP & $0.93 \pm 0.10$ \\
\hline E407Q & TPP & $1.65 \pm 0.48$ \\
\hline D411N & TPP & - \\
\hline WT & $\mathrm{Pm}$ & $1 \pm 0.17$ \\
\hline D34N & $\mathrm{Pm}$ & - \\
\hline D61N & $\mathrm{Pm}$ & $0.90 \pm 0.20$ \\
\hline D323N & $\mathrm{Pm}$ & - \\
\hline E406Q & $\mathrm{Pm}$ & $1 \pm 0.13$ \\
\hline E407Q & $\mathrm{Pm}$ & $0.58 \pm 0.2$ \\
\hline D411N & $\mathrm{Pm}$ & - \\
\hline WT & $\mathrm{Dq}$ & $0.90 \pm 0.21$ \\
\hline D34N & $\mathrm{Dq}$ & - \\
\hline D61N & $\mathrm{Dq}$ & $0.79 \pm 0.17$ \\
\hline D323N & $\mathrm{Dq}$ & $0.50 \pm 0.10$ \\
\hline E406Q & $\mathrm{Dq}$ & $0.87 \pm 0.3$ \\
\hline E407Q & $\mathrm{Dq}$ & - \\
\hline D411N & $\mathrm{Dq}$ & $0.71 \pm 0.2$ \\
\hline
\end{tabular}


Table 2

Experimental observations are summarised in the table below.

\begin{tabular}{|c|c|c|c|c|c|c|c|c|c|c|}
\hline \multirow[t]{2}{*}{ Mutants } & \multicolumn{3}{|c|}{ Binding } & \multicolumn{3}{|c|}{ Transport } & \multicolumn{3}{|c|}{ Proton release } & \multirow[t]{2}{*}{ Remarks } \\
\hline & TPP & Pm & Dq & TPP & Pm & Dq & TPP & Pm & Dq & \\
\hline WT & $\checkmark$ & $\checkmark$ & $\checkmark$ & $\checkmark$ & $\checkmark$ & $\checkmark$ & ++ & +++ & +++ & $\begin{array}{l}\text { Neutral mutants of acidic residues present in the transport } \\
\text { vestibule of QacA are compared w.r.t WT }\end{array}$ \\
\hline \multirow[t]{2}{*}{ D34N } & & & & & & & & & & ESSENTIAL \\
\hline & $\mathbf{x}$ & $\mathbf{x}$ & $\mathbf{x}$ & $\mathbf{x}$ & $\mathbf{x}$ & $\mathbf{x}$ & - & + & + & $\begin{array}{l}\text { D34 is an essential residue required for recognition and } \\
\text { transport of all three substrates tested }\end{array}$ \\
\hline \multirow[t]{2}{*}{ D61N } & $\checkmark$ & $\checkmark$ & $\checkmark$ & $\checkmark$ & $\checkmark$ & $\checkmark$ & +++ & ++ & ++ & NON-ESSENTIAL \\
\hline & & & & & & & Tוד & Tा & TT & D61 is not a crucial residue for transport activity of QacA \\
\hline \multirow[t]{2}{*}{ D323N } & & & & & & & & & & CONDITIONAL \\
\hline & $\checkmark$ & $\mathbf{x}$ & $\checkmark$ & $\checkmark$ & $\mathbf{x}$ & $\checkmark$ & ++ & + & ++ & $\begin{array}{l}\text { D } 323 \text { is essential for Pm recognition whereas it is non-essential } \\
\text { for TPP and Dq transport }\end{array}$ \\
\hline \multirow[t]{2}{*}{ E406Q } & $r$ & $\checkmark$ & $\checkmark$ & $\checkmark$ & $r$ & $\checkmark$ & t+t & t+ & t+ & NON-ESSENTIAL \\
\hline & . & 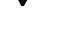 & 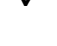 & 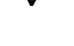 & 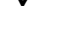 & . & +++ & ++ & ++ & E406 is not an essential residue for transport activityof QacA \\
\hline \multirow[t]{2}{*}{ E407Q } & & & & & & & & & & ESSENTIAL \\
\hline & $\checkmark$ & $\checkmark$ & $\mathbf{x}$ & $\checkmark$ & $\checkmark$ & $\mathbf{x}$ & + & ++ & + & $\begin{array}{l}\text { E407 is essential for Dq recognition whereas likely acting as } \\
\text { protonation site needed for TPP and Pm transport }\end{array}$ \\
\hline \multirow[t]{2}{*}{ D411N } & & & & & & & & & & CONDITIONAL \\
\hline & $\mathbf{x}$ & $x$ & $\checkmark$ & $\mathbf{x}$ & $\mathbf{x}$ & $\checkmark$ & - & + & +++ & $\begin{array}{l}\text { D411 is crucial for TPP and Pm recognition but not essential to } \\
\text { transport Dq }\end{array}$ \\
\hline
\end{tabular}

Note. $\checkmark$ represents binding and transport activity and $\mathbf{x}$ represents lack thereof; number of ' + ' represents number of proton release steps upon substrate addition whereas '-' represents no detectable release of protons as substrates are added. 